\title{
A FORMALITY THEOREM FOR POISSON MANIFOLDS
}

\author{
by
}

Gregory Ginot \& Gilles Halbout

\begin{abstract}
Let $M$ be a differential manifold. Using different methods, Kontsevich and Tamarkin have proved a formality theorem, which states the existence of a Lie homomorphism "up to homotopy" between the Lie algebra of Hochschild cochains on $C^{\infty}(M)$ and its cohomology $\left(\Gamma(M, \Lambda T M),[-,-]_{S}\right)$. Suppose $M$ is a Poisson manifold equipped with a Poisson tensor $\pi$; then one can deduce from this theorem the existence of a star product $\star$ on $C^{\infty}(M)$. In this paper we prove that the formality theorem can be extended to a Lie homomorphism "up to homotopy" between the Lie algebra of Hochschild cochains on the deformed algebra $\left(C^{\infty}(M), *\right)$ and the Poisson complex $\left(\Gamma(M, \Lambda T M),[\pi,-]_{S}\right)$. We will first recall Tamarkin's proof and see how the formality maps can be deduced from Etingof-Kazhdan's theorem using only homotopies formulas. The formality theorem for Poisson manifolds will then follow.
\end{abstract}

\section{0 . Introduction}

Let $M$ be a differential manifold. Formality theorems link commutative objects with non-commutative ones. More precisely, one can define two graded Lie algebras $\mathfrak{g}_{1}$ and $\mathfrak{g}_{2}$. The first one $\mathfrak{g}_{1}=\Gamma(M, \Lambda T M)$ is the space of multivector fields on $M$. It is endowed with a graded Lie bracket $[-,-]_{S}$ called the Schouten bracket (see $[\mathbf{K o s}]$ ).

The space $\mathfrak{g}_{1}$ can be identified with the cohomology of a cochain complex $\mathfrak{g}_{2}=$ $C(A, A)=\bigoplus_{k \geq 0} C^{k}(A, A)$, the space of Hochschild cochains (generated by differential $k$-linear maps from $A^{k}$ to $A$ ), where $A=C^{\infty}(M)$ is the algebra of smooth differential functions over $M$. The vector space $\mathfrak{g}_{2}$ is also endowed with a graded Lie algebra structure given by the Gerstenhaber bracket $[-,-]_{G}[\mathbf{G V}]$. The differential $b=$ $[m,-]_{G}$ (where $m \in C^{2}(A, A)$ is the commutative multiplication in $A$ ) makes $\mathfrak{g}_{2}$ into a graded differential Lie algebra and the cohomology $H^{*}\left(\mathfrak{g}_{2}, b\right)$ of $\mathfrak{g}_{2}$ with respect to $b$

2000 Mathematics Subject Classification. - Primary 16E40, 53D55, Secondary 18D50, 16S80. Key words and phrases. - Deformation quantization, star-product, homotopy formulas, homological methods. 
coincides with $\mathfrak{g}_{1}$. More precisely, one can construct a quasi-isomorphism $\phi^{1}: \mathfrak{g}_{1} \rightarrow$ $\mathfrak{g}_{2}$, (the Hochschild-Kostant-Rosenberg quasi-isomorphism, see [HKR]) between the complexes $\left(\mathfrak{g}_{1}, 0\right)$ and $\left(\mathfrak{g}_{2}, b\right)$; it is defined, for $\alpha \in \mathfrak{g}_{1}, f_{1}, \cdots, f_{n} \in A$, by

$$
\alpha \mapsto\left(\left(f_{1}, \ldots, f_{n}\right) \mapsto\left\langle\alpha, d f_{1} \wedge \cdots \wedge d f_{n}\right\rangle\right) .
$$

This map $\phi^{1}$ is not a Lie algebra morphism, but the only obstructions for it to be are given by boundaries for the differential $b$. In fact, Kontsevich's formality theorem states that $\phi^{1}$ induces a morphism if one relaxes the Lie algebras structures on $\mathfrak{g}_{1}$ and $\mathfrak{g}_{2}$ into Lie algebras "up to homotopy" structures. In other words, setting $\phi^{0}=m \in C^{2}(A, A)$, formality theorems can be seen as a construction of a collection of homotopies $\phi^{n}: \Lambda^{n} \mathfrak{g}_{1} \rightarrow \mathfrak{g}_{2}$ such that $\phi^{1}$ is the Hochschild-Kostant-Rosenberg morphism and the map $\phi=\sum_{n \geq 0} \phi^{n}: \Lambda \cdot \mathfrak{g}_{1} \rightarrow \mathfrak{g}_{2}$ satisfies

$$
[\phi, \phi]_{G}=\phi \circ m_{1}^{1,1}
$$

where $m_{1}^{1,1}: \Lambda \mathfrak{g}_{1} \rightarrow \Lambda \cdot \mathfrak{g}_{1}$ is the canonical extension of the Schouten Lie bracket on $\mathfrak{g}_{1}$.

The existence of such homotopies was proven by Kontsevich (see [Ko1] and [Ko2]) and Tamarkin (see $[\mathbf{T a}]$ ). They use different methods in their proofs. Nevertheless the two approaches are connected (see [KS]). In this paper we will use Tamarkin's methods (which are also well explained in [Hi]) to obtain a version of the formality theorem when the manifold $M$ is equipped with a Poisson structure. Moreover, as in Tamarkin's proof, we will suppose that $M=\mathbb{R}^{n}$. In some cases the results can be globalized using techniques of Cattaneo, Felder and Tomassini (see [CFT]). More precisely, all our results are valid for a arbitrary manifolds up to Section 5 where acyclicity of the de Rham complex only holds for $M=\mathbb{R}^{n}$ and thus globalization is needed.

One of the goals of this paper is to make the maps $\phi^{n}$ given by Tamarkin's proof as explicit as possible. We could try to construct them by induction starting from $\phi^{1}$ (the Hochschild-Kostant-Rosenberg quasi-isomorphism), but we would meet cohomological obstructions to build $\left(\phi^{n}\right)_{n \geq 2}$. Thus a natural idea consists in enlarging the structures in order to reduce the obstructions. More precisely, we know that the graded space $\mathfrak{g}_{1}=\Gamma(M, \Lambda T M)$ equipped with the Lie bracket $[-,-]_{S}$ and the exterior product $\wedge$ has a graded Gerstenhaber algebra structure.

Although the complex $\mathfrak{g}_{2}$ equipped with the Gerstenhaber bracket and the cupproduct is not a Gerstenhaber algebra, Tamarkin [Ta] has proved that $\mathfrak{g}_{2}$ can be endowed with a structure of Gerstenhaber algebras up to homotopy (see Section 1) and established the existence of a quasi-isomorphism of Gerstenhaber algebras up to homotopy between $\mathfrak{g}_{1}$ and $\mathfrak{g}_{2}$.

The paper is organized as follows:

- In Section 1, taking our inspiration from the language of operads, we recall the definitions of Lie algebras up to homotopy $\left(L_{\infty}\right.$-algebras for short) and reformulate the problem as follows: the (differential) graded Lie algebra structure on $\mathfrak{g}_{1}$ and $\mathfrak{g}_{2}$ are equivalent to codifferentials $d_{1}$ and $d_{2}$ on the exterior coalgebras 
$\Lambda \mathfrak{g}_{1}$ and $\Lambda^{\prime} \mathfrak{g}_{2}$. A morphism of Lie algebras up to homotopy between $\mathfrak{g}_{1}$ and $\mathfrak{g}_{2}$ is a morphism of differential coalgebras

$$
\phi:\left(\Lambda \mathfrak{g}_{1}, d_{1}\right) \rightarrow\left(\Lambda \mathfrak{g}_{2}, d_{2}\right) .
$$

We will also recall the definition of Gerstenhaber algebras "up to homotopy" or $G_{\infty}$-algebras (similarly given by a differential $d$ on a peculiar coalgebra $\Lambda^{\prime} \underline{\mathfrak{g}^{\otimes \cdot} \text { ) }}$ and morphism between them.

- In Section 2 we recall Tamarkin's construction of the $G_{\infty}$-structure on $\mathfrak{g}_{2}$, given by a differential $d_{2}$ on $\Lambda \cdot \mathfrak{g}_{2}^{\otimes}$.

- In Section 3 we prove (still following Tamarkin's approach) that there exists a $G_{\infty}$-structure on $\mathfrak{g}_{1}$, given by a differential $d_{1}^{\prime}$ on $\Lambda \mathfrak{g}_{1}^{\otimes}$, and that there exists a $G_{\infty}$-morphism $\psi:\left(\Lambda \underline{\mathfrak{g}_{1}^{\otimes}}, d_{1}^{\prime}\right) \rightarrow\left(\Lambda \underline{\mathfrak{g}_{2}^{\otimes}}, d_{2}\right)$.

- In Section 4 we establish the existence of a $G_{\infty}$-morphism $\psi^{\prime}:\left(\Lambda^{\cdot} \underline{\mathfrak{g}_{1}^{\otimes}}, d_{1}\right) \rightarrow$ $\left(\Lambda \mathfrak{g}_{1}^{\otimes \cdot}, d_{1}^{\prime}\right)$, where $d_{1}$ defines the Gerstenhaber structure on $\mathfrak{g}_{1}$ described in Section 1 . We deduce this fact from the acyclicity of the complex

$$
\left(\operatorname{Hom}\left(\Lambda \underline{\mathfrak{g}_{1}^{\otimes} \cdot}\right),\left[m_{1}^{1,1}+m_{1}^{2},-\right]\right),
$$

where $[-,-]$ denotes the graded commutator of morphisms,.

- In Section 5 we prove that the complex $\left(\operatorname{Hom}\left(\Lambda \underline{\mathfrak{g}}_{1}^{\otimes \cdot}\right),\left[m_{1}^{1,1}+m_{1}^{2},-\right]\right)$ is acyclic for $M=\mathbb{R}^{n}$ and that homotopy formulas can be written out.

- In Section 6 we show that the $G_{\infty}$-morphism $\phi=\psi \circ \psi^{\prime}:\left(\Lambda \cdot \mathfrak{g}_{1}^{\otimes \cdot}, d_{1}\right) \rightarrow$ $\left(\Lambda \mathfrak{g}_{2}^{\otimes \cdot}, d_{2}\right)$ induces the desired $L_{\infty}$-morphism between $\mathfrak{g}_{1}$ and $\mathfrak{g}_{2}$ and also in the same way an associative algebra morphism "up to homotopy" between these two spaces. Moreover when the manifold $M$ is a Poisson manifold equipped with a Poisson tensor field $\pi \in \Gamma\left(M, \Lambda^{2} T M\right)$ satisfying $[\pi, \pi]_{S}=0$, then there exists a star-product on $M$, that is to say a deformation $m_{\star}$ of the product $m$ on $A$ whose linear term is $\pi$ (see [BFFLS1] and [BFFLS2]). In this case $\left(\mathfrak{g}_{1},[-,-]_{S},[\pi,-]_{S}\right)$ becomes a graded differential Lie algebra (and even a Gerstenhaber algebra) and $\left(\mathfrak{g}_{2},[-,-]_{G}, b_{\star}\right)$, where $b_{\star}$ is the Hochschild differential corresponding to the deformed product $m_{\star}$, is a new graded differential Lie algebra.

- In Section 7 we prove a formality theorem between the two differential graded Lie algebras $\left(\mathfrak{g}_{1 \star},[-,-]_{S},[\pi,-]_{S}\right)$ and $\left(\mathfrak{g}_{2 \star},[-,-]_{G}, b_{\star}\right)$ following the same steps as in Sections 2, 3 and 4.

Remark: In this paper we emphasize the Lie structures of $\mathfrak{g}_{1}$ and $\mathfrak{g}_{2}$, not their associative algebra structures. Hence, our gradings for the spaces $\mathfrak{g}_{1}, \mathfrak{g}_{2}, \ldots$ are shifted by one from what is usually done in the literature.

\section{Definitions and notations}

Let $\mathfrak{g}$ be any graded vector space. The exterior coalgebra $\Lambda \mathfrak{g}$ is the cofree commutative coalgebra on the vector space $\mathfrak{g}$. In this paper we deal with graded space. 
Henceforth, for any graded vector space $\mathfrak{g}$, we choose the following degree on $\Lambda \mathfrak{g}:$ if $X_{1}, \ldots, X_{k}$ are homogeneous elements of respective degree $\left|X_{1}\right|, \ldots\left|X_{k}\right|$, then

$$
\left|X_{1} \wedge \cdots \wedge X_{k}\right|=\left|X_{1}\right|+\cdots+\left|X_{k}\right|-k .
$$

In particular the component $\mathfrak{g}=\Lambda^{1} \mathfrak{g} \subset \Lambda_{\mathfrak{g}} \mathfrak{g}$ is the same as the Lie algebra $\mathfrak{g}$ with degree shifted by one. The coalgebra $\Lambda \cdot \mathfrak{g}$ being cofree, any degree one map $d^{k}: \Lambda^{k} \mathfrak{g} \rightarrow$ $\mathfrak{g}(k \geq 1)$ extends into a derivation $d^{k}: \Lambda \cdot \mathfrak{g} \rightarrow \Lambda \cdot \mathfrak{g}$ of the coalgebra $\Lambda \cdot \mathfrak{g}$.

Let us recall the definition of Lia algebras "up to homotopy", denoted $L_{\infty}$-algebras henceforth.

Definition 1.1. - A vector space $\mathfrak{g}$ is endowed with a $L_{\infty}$-algebra structure if there are degree one linear maps $m^{1, \ldots, 1}: \Lambda^{k} \mathfrak{g} \rightarrow \mathfrak{g}$ such that if we extend them to maps $\Lambda \mathfrak{g} \rightarrow \Lambda \cdot \mathfrak{g}$, then $d \circ d=0$ where $d$ is the derivation

$$
d=m^{1}+m^{1,1}+\cdots+m^{1, \ldots, 1}+\cdots .
$$

For more details on $L_{\infty}$-structures, see [LS]. It follows from the definition that a $L_{\infty}$-algebra structure induces a differential coalgebra structure on $\Lambda \cdot \mathfrak{g}$ and that the map $m^{1}: \mathfrak{g} \rightarrow \mathfrak{g}$ is a differential.

The Lie algebra structure on $\mathfrak{g}_{1}=\Gamma(M, \Lambda T M)$ is given by the Schouten bracket (see [Kos]) which extends the Lie bracket of vector fields in the following way:

$$
[\alpha, \beta \wedge \gamma]_{S}=[\alpha, \beta]_{S} \wedge \gamma+(-1)^{|\alpha|(|\beta|+1)} \beta \wedge[\alpha, \gamma]_{S}
$$

for $\alpha, \beta, \gamma \in \mathfrak{g}_{1}$. For $f \in \Gamma\left(M, \Lambda^{0} T M\right)=C^{\infty}(M)$ and $\alpha \in \Gamma\left(M, \Lambda^{1} T M\right)$ we set $[\alpha, f]_{S}=\alpha \cdot f$, the action of the vector field $\alpha$ on $f$. The grading on $\mathfrak{g}_{1}$ is defined by $|\alpha|=n \Leftrightarrow \alpha \in \Gamma\left(M, \Lambda^{n+1} T M\right)$

We reformulate the graded Lie algebra structure of $\mathfrak{g}_{1}$ into a $L_{\infty}$-algebra structure as follows: the Schouten Lie bracket $[-,-]_{S}$ on $\mathfrak{g}_{1}$ is equivalent to a (degree one) map $m_{1}^{1,1}: \Lambda^{2} \mathfrak{g}_{1} \rightarrow \mathfrak{g}_{1}$ that we can extend canonically to $m_{1}^{1,1}: \Lambda \mathfrak{g}_{1} \rightarrow \Lambda \cdot \mathfrak{g}_{1}$. The Jacobi identity satisfied by $[-,-]_{S}$ then corresponds to the identity:

$$
d_{1} \circ d_{1}=0,
$$

where $d_{1}=m_{1}^{1,1}$. Hence the map $m_{1}^{1,1}$ defines a $L_{\infty}$-algebra structure on $\mathfrak{g}_{1}$.

In the same way, the Lie algebra structure on the vector space $\mathfrak{g}_{2}=C(A, A)$ is given by the Gerstenhaber bracket $[-,-]_{G}$ defined, for $D, E \in \mathfrak{g}_{2}$, by

$$
[D, E]_{G}=\{D \mid E\}-(-1)^{|E||D|}\{E \mid D\},
$$

where

$$
\{D \mid E\}\left(x_{1}, \ldots, x_{d+e-1}\right)=\sum_{i \geq 0}(-1)^{|E| \cdot i} D\left(x_{1}, \ldots, x_{i}, E\left(x_{i+1}, \ldots, x_{i+e}\right), \ldots\right) .
$$

The space $\mathfrak{g}_{2}$ has a grading defined by $|D|=k \Leftrightarrow D \in C^{k+1}(A, A)$ and its differential is $b=[m,-]_{G}$, where $m \in C^{2}(A, A)$ is the commutative multiplication on $A$.

The Gerstenhaber bracket on $C(A, A)$ is equivalent to a map $m_{2}^{1,1}: \Lambda^{2} \mathfrak{g}_{2} \rightarrow \mathfrak{g}_{2}$ and the differential $b$ is a degree one map $m_{2}^{1}: \mathfrak{g}_{2} \rightarrow \mathfrak{g}_{2}$. These maps extends to maps $\Lambda \mathfrak{g}_{2} \rightarrow \Lambda \mathfrak{g}_{2}$. All identities defining the differential Lie algebra structure on 
$\mathfrak{g}_{2}$ (Jacobi relations for $[-,-]_{G}, b^{2}=0$, compatibility between $b$ and $[-,-]_{G}$ ) can be summarized in the unique relation

$$
d_{2} \circ d_{2}=0
$$

where $d_{2}=m_{2}^{1}+m_{2}^{1,1}$. Hence the maps $b$ and $[-,-]_{G}$ defines a $L_{\infty}$-structure on $\mathfrak{g}_{2}$. In fact any differential Lie algebra $(\mathfrak{g}, b)$ has a $L_{\infty}$-structure, with $m_{2}^{1}=b, m_{2}^{1,1}$ is given by its bracket and $m^{1, \ldots, 1}: \Lambda^{k \geq 3} \mathfrak{g} \rightarrow \mathfrak{g}=0$.

Definition 1.2. - A $L_{\infty}$-morphism between two $L_{\infty}$-algebras $\left(\mathfrak{g}_{1}, d_{1}=m_{1}^{1}+\ldots\right)$ and $\left(\mathfrak{g}_{2}, d_{2}=m_{2}^{1}+\ldots\right)$ is a morphism of differential coalgebras

$$
\phi:\left(\Lambda \cdot \mathfrak{g}_{1}, d_{1}\right) \rightarrow\left(\Lambda \mathfrak{g}_{2}, d_{2}\right)
$$

Such a map $\phi$ is uniquely determined by a collection of maps $\phi^{n}: \Lambda^{n} \mathfrak{g}_{1} \rightarrow \mathfrak{g}_{2}$ as the differential coalgebras $\Lambda \mathfrak{g}_{1}$ and $\Lambda \mathfrak{g}_{2}$ are cofree. In the case $\mathfrak{g}_{1}$ and $\mathfrak{g}_{2}$ are respectively the graded Lie algebra $\left(\Gamma(M, \Lambda T M),[-,-]_{S}\right)$ and the differential graded Lie algebra $\left(C\left(C^{\infty}(M), C^{\infty}(M)\right),[-,-]_{G}\right)$ it is easy to check that Definition (0.1) (from the introduction) and Definition (1.3) coincide.

A shuffle (of length $n$ ) is a permutation of $\{1, \ldots, n\}(n \geq 1)$ such that there exist $p, q \geq 1$ with $p+q=n$ and the following inequalities hold:

$$
\sigma(1)<\cdots<\sigma(p), \quad \sigma(p+1)<\cdots<\sigma(p+q) .
$$

For any permutation $\sigma$ of $\{1, \ldots, n\}$ and any graded variables $x_{1}, \ldots, x_{n}$ we define the sign $\varepsilon(\sigma)$ (the dependance on $x_{1}, \ldots x_{n}$ is implicit) by the identity

$$
x_{1} \ldots x_{n}=\varepsilon(\sigma) x_{\sigma^{-1}(1)} \ldots x_{\sigma^{-1}(n)}
$$

which holds in the free graded commutative algebra generated by $x_{1}, \ldots x_{n}$.

For any graded vector space $\mathfrak{g}$, each shuffle $\sigma$ acts on $\mathfrak{g}^{\otimes n}$ by the formula:

$$
\sigma \cdot\left(a_{1} \otimes \cdots \otimes a_{n}\right)=(-1)^{\sigma} \varepsilon(\sigma) a_{\sigma^{-1}(1)} \otimes \cdots a_{\sigma^{-1}(n)}
$$

for $a_{0}, \cdots, a_{n} \in \mathfrak{g}$. We denote $\mathfrak{g}^{\otimes n}$ the quotient of $\mathfrak{g}^{\otimes n}$ by the image of all the maps $\operatorname{shuf}_{p, q}=\sum \sigma \cdot(-)$, where the sum is over all shuffles of length $n=p+q$ with $p, q$ fixed. The graded vector space $\oplus_{n \geq 0} \mathfrak{g}^{\otimes n}$ a quotient coalgebra of the tensor coalgebra $\oplus_{n \geq 0} \mathfrak{g}^{\otimes n}$. It is well known (see [GKK] for example) that this coalgebra $\oplus_{n \geq 0} \mathfrak{g}^{\otimes n}$ is the cofree Lie coalgebra on the vector space $\mathfrak{g}$ (with degree shifted by minus one).

Henceforth, for any space $\mathfrak{g}$, we denote $\Lambda \cdot \underline{\mathfrak{g}}^{\otimes}$ the graded space $\underset{m \geq 1, p_{1}+\cdots+p_{n}=m}{\oplus} \underline{\mathfrak{g}^{\otimes p_{1}}} \wedge$ $\cdots \wedge \underline{\mathfrak{g}^{\otimes p_{n}}}$. We will use the following grading on $\Lambda \mathfrak{g}^{\otimes \cdot}:$ for $x_{1}^{1}, \cdots, x_{n}^{p_{n}} \in \mathfrak{g}$, we define

$$
\underline{\mid x_{1}^{1} \otimes \cdots \otimes x_{1}^{p_{1}}} \wedge \cdots \wedge \underline{x_{n}^{1} \otimes \cdots \otimes x_{n}^{p_{n}} \mid}=\sum_{i_{1}}^{p_{1}}\left|x_{1}^{i_{1}}\right|+\cdots+\sum_{i_{n}}^{p_{1}}\left|x_{n}^{i_{n}}\right|-n .
$$

Notice that the induced grading on $\Lambda \mathfrak{g} \subset \Lambda \mathfrak{g}^{\otimes \cdot}$ is the same than the one introduced above. The cobracket on $\oplus \underline{\mathfrak{g}^{\otimes}}$ and the coproduct on $\Lambda \mathfrak{g}$ extend to a cobracket and a coproduct on $\Lambda \mathfrak{g}^{\otimes}$. The sum of the cobracket and the coproduct give rise to a coalgebra structure on $\Lambda \mathfrak{g}^{\otimes \cdot}$. It is well known that this coalgebra structure is cofree (see [Gi],Section 3 for example). 
Definition 1.3. - A structure of Gerstenhaber algebra "up to homotopy" ( $G_{\infty}$ algebra for short) on a graded vector space $\mathfrak{g}$ is given by a collection of degree one maps

$$
m^{p_{1}, \ldots, p_{n}}: \underline{\mathfrak{g}^{\otimes p_{1}}} \wedge \cdots \wedge \underline{\mathfrak{g}^{\otimes p_{n}}} \rightarrow \mathfrak{g}
$$

indexed by $p_{1}, \ldots p_{n} \geq 1$ such that their canonical extension: $\Lambda \underline{\mathfrak{g}^{\otimes}} \rightarrow \Lambda \underline{\mathfrak{g}^{\otimes \cdot}}$ satisfies $d \circ d=0$ where

$$
d=\sum_{m \geq 1, p_{1}+\cdots p_{n}=m} m^{p_{1}, \ldots, p_{n}}
$$

More details on $G_{\infty}$-structures are given in [Gi]. Again, as the coalgebra structure of $\Lambda \cdot \underline{\mathfrak{g}^{\otimes}}$ is cofree, the map $d$ makes $\Lambda \underline{\mathfrak{g}^{\otimes}}$ a differential coalgebra.

Definition 1.4. - A morphism of $G_{\infty}$-algebras between two $G_{\infty}$-algebras $\left(\mathfrak{g}_{1}, d_{1}\right)$ and $\left(\mathfrak{g}_{2}, d_{2}\right)$ is a map $\phi:\left(\Lambda \underline{\mathfrak{g}_{1}^{\otimes \cdot}}, d_{1}\right) \rightarrow\left(\Lambda \underline{\mathfrak{g}_{2}^{\otimes \cdot}}, d_{2}\right)$ of codifferential coalgebras.

The Lie algebra $\mathfrak{g}_{1}$ of multivectorfields is in fact a Gerstenhaber algebra that is to say a graded Lie algebra structure with a graded commutative algebra structure (for the same space with grading shifted by -1 ) and a compatibility between the bracket and the product (expressing that the bracket is a derivation for the product) as in (1.2). On the space $\mathfrak{g}_{1}=\Gamma(M, \Lambda T M)$, the commutative structure is given by the exterior product:

$$
\forall \alpha, \beta \in \Gamma(M, \Lambda T M), \alpha \wedge \beta=(-1)^{(|\alpha|+1)(|\beta|+1)} \beta \wedge \alpha .
$$

We can reformulate the graded Gerstenhaber structure into a $G_{\infty}$-algebra structure as follows. The graded Lie algebra structure is still given by a map $m_{1}^{1,1}: \Lambda^{2} \mathfrak{g}_{1} \rightarrow \mathfrak{g}_{1}$, and the commutative graded algebra structure is given by a map $m_{1}^{2}: \mathfrak{g}_{1}^{\otimes 2} \rightarrow \mathfrak{g}_{1}$ (because $\mathfrak{g}_{1}^{\otimes 2}$ is the quotient of $\mathfrak{g}_{1}^{\otimes 2}$ by the 2 -shuffles, that is to say the elements $a \otimes b-\left(\overline{-1)^{(}}(a \mid+1)(|b|+1) b \otimes a\right)$. The maps $m_{1}^{1,1}, m_{1}^{2}$ above extend into degree one derivations

$$
m_{1}^{1,1}, m_{1}^{2}: \wedge \underline{\mathfrak{g}_{1}^{\otimes}} \rightarrow \wedge \underline{\mathfrak{g}_{1}^{\otimes}} .
$$

All the identities defining the Gerstenhaber-algebra structure on $\mathfrak{g}_{1}$ can be summarized into the unique relation

$$
d_{1} \circ d_{1}=0
$$

where $d_{1}=m_{1}^{1,1}+m_{1}^{2}$. Hence the Gerstenhaber bracket and the exterior product define a $G_{\infty}$-algebra structure on $\mathfrak{g}_{1}$. More generally, any Gerstenhaber algebra $(\mathfrak{g}, m,[-,-])$ has a canonical $G_{\infty}$-structure given by $m^{2}=m, m^{1,1}=[-,-]$, the other maps being zero. 


\section{A $G_{\infty}$-structure on $\mathfrak{g}_{2}=C(A, A)$}

The Lie algebra $\mathfrak{g}_{2}$ is also endowed with an associative product. It is the "cup" product $\cup$ defined, for $D, E \in \mathfrak{g}_{2}$ and $x_{1}, \ldots, x_{|D|+|E|+2} \in A$, by

$$
(D \cup E)\left(x_{1}, \ldots, x_{|D|+|E|+2}\right)=(-1)^{\gamma} D\left(x_{1}, \ldots, x_{|D|+1}\right) E\left(x_{|D|+2}, \ldots, x_{|D|+|E|+2}\right)
$$

where $\gamma=(|E|+1)(|D|+1)$. The projection of this product on the cohomology of $\left(\mathfrak{g}_{2}, b\right)$ is the exterior product $\wedge$, but unfortunately $\left(\mathfrak{g}_{2},[-,-]_{G}, \cup, b\right)$ is not a Gerstenhaber algebra. However the relations (1.2), (1.4) are satisfied up to a boundary for $b$.

Tamarkin stated the existence of a $G_{\infty}$-structure on $\mathfrak{g}_{2}$. Our aim in this section is to build this $G_{\infty}$-structure more explicitly. By Definition 1.3. we have to exhibit a differential $d_{2}$ on $\Lambda \underline{\mathfrak{g}}_{2}^{\otimes}$ satisfying, if

$$
d_{2}=m_{2}^{1}+m_{2}^{1,1}+m_{2}^{2}+\cdots+m_{2}^{p_{1}, \ldots, p_{n}}+\cdots,
$$

1. $m_{2}^{1}$ is the map $b$ and $m_{2}^{1,1}$ is the map $[-,-]_{G}$.

2. $d_{2} \circ d_{2}=0$.

We first reformulate this problem: let $L_{2}=\oplus \mathfrak{g}_{2}^{\otimes n}$ be the cofree Lie coalgebra on $\mathfrak{g}_{2}$ (see Section 1 for the notation). Since $L_{2}$ is a cofree coalgebra, a Lie bialgebra structure on $L_{2}$ is given by degree one maps $l_{2}^{n}: \mathfrak{g}_{2}^{\otimes n} \rightarrow \mathfrak{g}_{2}$, corresponding to the differential, and maps $l_{2}^{p_{1}, p_{2}}: \mathfrak{g}_{2}^{\otimes p_{1}} \wedge \mathfrak{g}_{2}^{\otimes p_{2}} \rightarrow \mathfrak{g}_{2}$, corresponding to the Lie bracket. These maps extend uniquely into a coalgebra derivation $L_{2} \rightarrow L_{2}$ and a coalgebra map $L_{2} \wedge L_{2} \rightarrow L_{2}$ (still denoted $l_{2}^{m}$ and $l_{2}^{p, q}$ ). The following lemma is well known.

Lemma 2.1. - Suppose we have a differential Lie bialgebra structure on the Lie coalgebra $L_{2}$, with differrential and Lie bracket respectively determined by maps $l_{2}^{n}$ and $l_{2}^{p_{1}, p_{2}}$ as above. Then $\mathfrak{g}_{2}$ has a $G_{\infty}$-structure given, for all $p, q, n \geq 1$, by

$$
m_{2}^{n}=l_{2}^{n}, \quad m_{2}^{p, q}=l_{2}^{p, q} \quad \text { and } \quad m_{2}^{p_{1}, \ldots, p_{r}}=0 \text { for } r \geq 3 .
$$

Proof: The map $d_{2}=\sum_{i \geq 0} l_{2}^{i}+\sum_{p_{1}, p_{2} \geq 0} l_{2}^{p_{1}, p_{2}}: \Lambda^{\prime} L_{2} \rightarrow \Lambda^{\prime} L_{2}$ is the ChevalleyEilenberg differential on the differential Lie algebra $L_{2}$; it satisfies $d_{2} \circ d_{2}=0$.

Thus to obtain the desired $G_{\infty}$-structure on $\mathfrak{g}_{2}$, it is enough to define a Lie bialgebra structure on $L_{2}$ given by maps $l_{2}^{n}$ and $l_{2}^{p_{1}, p_{2}}$ with $l_{2}^{1}=b$ and $l_{2}^{1,1}=[-,-]_{G}$.

Let us now give an equivalent formulation of our problem, which is stated in terms of the associated operads in [Ta]:

Proposition 2.2. - Differential Lie bialgebra structures on the cofree Lie coalgebra $L_{2}=\oplus_{n \geq 0} \underline{\mathfrak{g}}_{2}^{\otimes n}$ are in one to one correspondence with differential bialgebra structures on the cofree tensorial coalgebra $T_{2}=\oplus_{n \geq 0} \mathfrak{g}_{2}^{\otimes n}$.

A differential bialgebra structure on the cofree tensorial coalgebra $\oplus V^{\otimes n}$ associated to a vector space $V$ is often called a $B_{\infty}$-structure on $V$, see [Ba].

Proof: We follow the proof in [Ta]. Let $V$ be a finite-dimensional vector space and $V^{*}$ be the dual space. A differential bialgebra structure on $T=\oplus_{n \geq 0} V^{\otimes n}$ is given by maps $a^{n}: V^{\otimes n} \rightarrow V(n \geq 2)$, corresponding to the differential, and maps $a^{p_{1}, p_{2}}$ : 
$V^{\otimes p_{1}} \otimes V^{\otimes p_{2}} \rightarrow V\left(p_{1}, p_{2} \geq 0\right)$, corresponding to the product. We can define dual maps of the maps $\sum_{n \geq 0} a^{n}: T \rightarrow T$ and $\sum_{p_{1}, p_{2} \geq 0} a^{p_{1}, p_{2}}: T \otimes T \rightarrow T$, namely $D: \hat{T} \rightarrow \hat{T}$ and $\Delta: \hat{T} \rightarrow \hat{T} \hat{\otimes} \hat{T}$, where $\hat{T}$ is the completion of the tensor algebra $\oplus_{n \geq 0} V^{* \otimes n}$. The maps $D$ and $\Delta$ are given by maps $a^{n *}: V^{*} \rightarrow V^{* \otimes n}$ and $a^{p_{1}, p_{2}{ }^{*}}:$ $V^{*} \rightarrow V^{* \otimes p_{1}} \otimes V^{* \otimes p_{2}}$, and define a differential bialgebra structure on the complete free algebra $\hat{T}$. The tensor algebra $\oplus_{n \geq 0} V^{* \otimes n}$ is now graded as follows: $|x|=p$ when $x \in V^{* \otimes p}$.

Similarly, if we consider a differential Lie bialgebra structure on the cofree Lie coalgebra $L=\oplus_{n \geq 0} \underline{V^{\otimes n}}$, the duals maps $d$ and $\delta$ of the structure maps $\sum_{n \geq 0} l^{n}$ and $\sum_{p_{1}, p_{2} \geq 0} l^{p_{1}, p_{2}}$ induce a differential Lie bialgebra structure on $\hat{L}$, the completion of the free Lie algebra $\oplus_{n \geq 0} \operatorname{Lie}\left(V^{*}\right)(n)$ on $V^{*}$, where $\operatorname{Lie}\left(V^{*}\right)(n)$ is the subspace of element of degree $n$.

We now replace formally each element $x$ of degree $n$ in $\hat{T}$ (resp. $\hat{L}$ ) by $h^{n} x$, where $h$ is a formal parameter. Letting $|h|=-1$, we easily see that a differential associative (resp. Lie) bialgebra structure on the associative (resp. Lie) algebras $\left(\oplus_{n \geq 0} V^{* \otimes n}\right)[[h]]$ (resp. $\left.\left(\oplus_{n \geq 0} \operatorname{Lie}\left(V^{*}\right)(n)\right)[[h]]\right)$ with the product and coproduct being of degree zero is equivalent to a differential associative (resp. Lie) bialgebra structure on the associative (resp. Lie) algebra $\hat{T}$ (resp. $\hat{L}$ ).

Suppose we have a differential free coalgebra $(\hat{T}, D, \Delta)$. We can apply EtingofKazhdan's dequantization theorem (see [EK2], Section 2.4): there exists a Lie bialgebra $\left(\hat{L}^{\prime},[-,-], \delta\right)$, generated as a Lie algebra by $V^{*}$ and an injective map $I_{\mathrm{EK}}$ : $\hat{L}^{\prime}[[h]] \rightarrow\left(\oplus_{n \geq 0} V^{* \otimes n}\right)[[h]]$ such that

1. the restriction $I_{\mathrm{EK}}: V^{*} \rightarrow V^{*}$ is the identity,

2. $I_{\mathrm{EK}}([a, b])=I_{\mathrm{EK}}(a) I_{\mathrm{EK}}(b)-I_{\mathrm{EK}}(b) I_{\mathrm{EK}}(a)$, for all $a, b \in \hat{L}^{\prime}$,

3. $\left(\Delta-\Delta^{\mathrm{op}}\right) I_{\mathrm{EK}}=h I_{\mathrm{EK}} \delta+O\left(h^{2}\right)$,

4. the maps $I_{\mathrm{EK}}, \delta$ and $[-,-]$ are given by universal formulas depending only on $\Delta$ and the product of $\hat{T}$,

5. if we apply Etingof-Kazhdan's quantization functor (see [EK1]) to the Lie bialgebra $\left(\oplus_{n \geq 0} \operatorname{Lie}\left(V^{*}\right)^{n}[[h]], \delta\right)$ we get the bialgebra $\left(\left(\oplus_{n \geq 0} V^{* \otimes n}\right)[[h]], \Delta\right)$ back.

The last condition implies that $\hat{L}^{\prime}$ is free as a Lie algebra because $\hat{T}$ is free as an algebra so that $\left(\hat{L}^{\prime}, d, \delta\right)$ is a differential free Lie coalgebra, taking $d$ such that $I_{\mathrm{EK}} \circ d=D \circ I_{\mathrm{EK}}$.

Starting now with a differential free Lie coalgebra $L$, we can use the same theorems to construct a differential free coalgebra such that this construction is the inverse of the previous one. This proves that differential Lie bialgebra structures on the free Lie coalgebra $\hat{L}$ and differential bialgebra structures on the free tensorial coalgebra $\hat{T}$ are in one to one correspondence and so, taking dual maps, differential Lie bialgebra structures on the cofree Lie coalgebra $L$ and differential bialgebra structures on the cofree tensorial coalgebra $T$ are also in one to one correspondence. Moreover, as the operations build in this correspondence are universal, depending only on the structure maps, the same assertion is still valid when $V$ is the infinite-dimensional space $\mathfrak{g}_{2}$.

Since the map $I_{\mathrm{EK}}$ defined in the precedent proof is the identity on $V^{*}$, the first terms $a_{2}^{1}$ and $l_{2}^{1}$ of the differentials will be the same on $T_{2}=\oplus \mathfrak{g}_{2}^{\otimes n}$ and on $L_{2}=$ 
$\oplus_{n \geq 0} \mathfrak{g}_{2}^{\otimes n} \mathfrak{g}_{2}^{*}$. For the same reason the first term $l_{2}^{1,1}$ of the Lie bracket on $L_{2}$ will be the antisymmetrization of the first term $a_{2}^{1,1}$ of the cobracket on $T_{2}$.

By Proposition 2.2, the problem of defining a Lie bialgebra structure on $L_{2}$ given by maps $l_{2}^{n}$ and $l_{2}^{p_{1}, p_{2}}$ with $l_{2}^{1}=b$ and $l_{2}^{1,1}=[-,-]_{G}$, is equivalent to defining a differential bialgebra structure on $T_{2}$ given by maps $a_{2}^{n}: \mathfrak{g}_{2}^{\otimes n} \rightarrow \mathfrak{g}_{2}$ and $a_{2}^{p_{1}, p_{2}}$ : $\mathfrak{g}_{2}^{\otimes p_{1}} \otimes \mathfrak{g}_{2}^{\otimes p_{2}} \rightarrow \mathfrak{g}_{2}$ where $a_{2}^{1}=b$ and $a_{2}^{1,1}$ is the product $\{-\mid-\}$ defined in Section 1 . Indeed, the anti-symmetrization of $\{-\mid-\}$ is by definition $[-,-]_{G}$. The latter can be achieved using the braces (defined in $[\mathbf{G V}]$ ) acting on the Hochschild cochain complex $\mathfrak{g}_{2}=C(A, A)$ for any algebra $A$. The braces operations are maps $a_{2}^{1, p}: \mathfrak{g}_{2} \otimes \mathfrak{g}_{2}^{\otimes p} \rightarrow \mathfrak{g}_{2}$ $(p \geq 1)$ defined, for all homogeneous $D, E_{1}, \ldots, E_{p} \in \mathfrak{g}_{2}^{\otimes p+1}$ and $x_{1}, \ldots, x_{d} \in A$ (with $\left.d=|D|+\left|E_{1}\right|+\cdots+\left|E_{p}\right|+1\right)$, by

$$
\begin{aligned}
& a_{2}^{1, p}\left(D_{1} \otimes\left(E_{1} \otimes \ldots \otimes E_{p}\right)\right)\left(x_{1} \otimes \cdots \otimes x_{d}\right)= \\
& \sum(-1)^{\tau} D\left(x_{1}, \ldots, x_{i_{1}}, E_{1}\left(x_{i_{1}+1}, \ldots\right), \ldots, E_{p}\left(x_{i_{p}+1}, \ldots\right), \ldots\right)
\end{aligned}
$$

where $\tau=\sum_{k=1}^{p} i_{k}\left(\left|E_{k}\right|+1\right)$. The maps $a_{2}^{1, p}: \mathfrak{g}_{2} \otimes \mathfrak{g}_{2}^{\otimes p} \rightarrow \mathfrak{g}_{2}$ and $a_{2}^{q \geq 2, p}=0$ give a unique bialgebra structure on the cofree cotensorial algebra $T_{2}=\oplus_{n \geq 0} \mathfrak{g}_{2}^{\otimes n}$. Similarly taking $a_{2}^{1}$ to be the Hochschild coboundary $b$ and $a_{2}^{2}$ to be the cup-product $\cup$, and $a_{2}^{q \geq 3}=0$, gives a unique differential bialgebra structure on the tensor coalgebra $T_{2}$. Theorem 3.1 in [Vo] asserts that these maps yield a differential bialgebra structure on the cofree coalgebra $T_{2}$ (the proof is a straightforward computation, also see [G V] and $[\mathbf{K h}])$.

Using this result, we can successively apply Proposition 2.2 and Lemma 2.1 to obtain the desired $G_{\infty}$-structure on $\mathfrak{g}_{2}$ given by maps $m_{2}^{p_{1}, \ldots, p_{k}}$ such that $m_{2}^{1}=b$ and $m_{2}^{1,1}=[-,-]_{G}$. By construction, the maps $m_{2}^{p_{1}, \ldots, p_{k}}$ are 0 for $k>2$. Moreover, the map $m_{2}^{2}$ coincides, up to a Hochschild coboundary, with the cup-product $\cup$ because, when passing to cohomology, they both give the same map $m_{1}^{2}$, corresponding to the product $\wedge$ of the Gerstenhaber algebra $\left(\mathfrak{g}_{1},[-,-]_{S}, \wedge\right)$.

$$
\text { 3. A } G_{\infty} \text {-morphism } \psi:\left(\Lambda \underline{\mathfrak{g}}_{1}^{\otimes}, d_{1}^{\prime}\right) \rightarrow\left(\Lambda \underline{\mathfrak{g}_{2}^{\otimes}}, d_{2}\right)
$$

The objective of this section is to prove the following proposition.

Proposition 3.1. - There exist a differential $d_{1}^{\prime}$ on $\Lambda \mathfrak{g}_{1}^{\otimes \cdot}$ and a morphism of differential coalgebras $\psi:\left(\Lambda \underline{\mathfrak{g}_{1}^{\otimes}}, d_{1}^{\prime}\right) \rightarrow\left(\Lambda \underline{\mathfrak{g}_{2}^{\otimes}}, d_{2}\right)$ such that the induced map $\psi^{1}: \mathfrak{g}_{1} \rightarrow \mathfrak{g}_{2}$ is the Hochschild-Kostant-Rosenberg map of Section 0.

Proof: For $i=1,2$ and $n \geq 0$, let us set

$$
V_{i}^{[n]}=\bigoplus_{p_{1}+\cdots+p_{k}=n} \underline{\mathfrak{g}_{i}^{\otimes p_{1}}} \wedge \cdots \wedge \underline{\mathfrak{g}_{i}^{\otimes p_{k}}}
$$


and $V_{i}^{[\leq n]}=\sum_{k \leq n} V_{i}^{[k]}$. Let $d_{2}^{p_{1}, \ldots, p_{k}}: \mathfrak{g}_{2}^{\otimes p_{1}} \wedge \cdots \wedge \mathfrak{g}_{2}^{\otimes p_{k}} \rightarrow \mathfrak{g}_{2}$ be the components

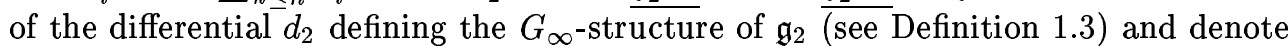
$d_{2}^{[n]}$ and $d_{2}^{[\leq n]}$ the sums

$$
d_{2}^{[n]}=\sum_{p_{1}+\cdots+p_{k}=n} d_{2}^{p_{1}, \ldots, p_{k}} \quad \text { and } \quad d_{2}^{[\leq n]}=\sum_{p \leq n} d_{2}^{[p]} .
$$

Clearly, $d_{2}=\sum_{n \geq 1} d_{2}^{[n]}$. In the same way, we denote

$$
d_{1}^{\prime[n]}=\sum_{p_{1}+\cdots+p_{k}=n} d_{1}^{p_{1}, \ldots, p_{k}} \quad \text { and } \quad d_{1}^{\prime[\leq n]}=\sum_{1 \leq k \leq n} d_{1}^{\prime^{[k]}} .
$$

We know from Section 1 that a morphism $\psi:\left(\Lambda \cdot \mathfrak{g}_{1}^{\otimes \cdot}, d_{1}^{\prime}\right) \rightarrow\left(\Lambda \mathfrak{g}_{2}^{\otimes \cdot}, d_{2}\right)$ is uniquely determined by its components $\psi^{p_{1}, \ldots, p_{k}}: \underline{\mathfrak{g}_{1}^{\otimes p_{1}}} \wedge \cdots \overline{\wedge \mathfrak{g}_{1}^{\otimes p_{k}}} \rightarrow \mathfrak{g}_{2}$. Similarly we set

$$
\psi^{[n]}=\sum_{p_{1}+\cdots+p_{k}=n} \psi^{p_{1}, \ldots, p_{k}} \quad \text { and } \quad \psi^{[\leq n]}=\sum_{1 \leq k \leq n} \psi^{[k]}
$$

Again, we have $d_{1}^{\prime}=\sum_{n \geq 1} d_{1}^{\prime^{[n]}}$ and $\psi=\sum_{n \geq 1} \psi^{[n]}$.

We have to build both the differential $d_{1}^{\prime}$ and the morphism of codifferential $\psi$. In fact we will build the maps $d_{1}^{\prime^{[n]}}$ and $\psi^{[n]}$ by induction. For the first terms, we set

$$
d_{1}^{[1]}=0 \quad \text { and } \quad \psi^{[1]}=\phi^{1},
$$

the Hochschild-Kostant-Rosenberg map (see Section 0).

Suppose we have built maps $\left(d_{1}^{\prime[i]}\right)_{i \leq n-1}$ and $\left(\psi^{[i]}\right)_{i \leq n-1}$ satisfying

$$
\psi^{[\leq n-1]} \circ d_{1}^{[\leq n-1]}=d_{2}^{[\leq n-1]} \circ \psi^{[\leq n-1]}
$$

on $V_{1}^{[\leq n-1]}$ and $d_{1}^{\prime[\leq n-1]} \circ d_{1}^{\prime[\leq n-1]}=0$ on $V_{1}^{[\leq n]}$. These conditions are enough to insure that $d_{1}^{\prime}$ is a differential and $\psi$ a morphism of differential coalgebras. If we reformulate the identity $\psi \circ d^{\prime}{ }_{1}=d_{2} \circ \psi$ on $V_{1}^{[n]}$, we get

$$
\psi^{[\leq n]} \circ d_{1}^{[\leq n]}=d_{2}^{[\leq n]} \circ \psi^{[\leq n]} .
$$

If we take now into account that $d_{1}^{d^{[1]}}=0$, and that on $V_{1}^{[n]}$ we have $\psi^{[k]} \circ d_{1}^{[l]}=$ $d_{2}^{[k]} \circ \psi^{[l]}=0$ for $k+l>n+1$, the identity (3.5) becomes

$$
\psi^{[1]} d_{1}^{[n]}+B=d_{2}^{[1]} \psi^{[n]}+A
$$

where $B=\sum_{k=2}^{n-1} \psi^{[\leq n-k+1]} d_{1}^{[k]}$ and $A=d_{2}^{[1]} \psi^{[\leq n-1]}+\sum_{k=2}^{n} d_{2}^{[k]} \psi^{[\leq n-k+1]}$ (we now omit the composition sign $\circ)$. The term $d_{2}^{[1]}$ in (3.6) is the Hochschild coboundary $b$. So thanks to the Hochschild-Kostant-Rosenberg theorem (3.6) is equivalent to the cochains $B-A$ being Hochschild cocycles. Therefore, in order to prove existence of $d_{1}^{\prime[n]}$ and $\psi^{[n]}$, it is sufficient to prove that

$$
d_{2}^{[1]}(B-A)=0
$$


and to show that for any choice of those maps, we have

$$
{d^{\prime}}_{1}^{[\leq n]} d_{1}^{[\leq n]}=0 \text { on } V_{1}^{[\leq n+1]} .
$$

- We will first construct $d_{1}^{\prime[2]}$ : for $n=2$, we get $A=d_{2}^{[1]} \psi^{[1]}+d_{2}^{[2]} \psi^{[1]}$ and $B=0$ so that

$$
\psi^{[1]} d_{1}^{[2]}=d_{2}^{[1]}\left(\psi^{[2]}+\psi^{[1]}\right)+d_{2}^{[2]} \psi^{[1]} .
$$

Thus $d_{1}^{[2]}$ is the image of $d_{2}^{[2]}$ through the projection on the cohomolgy of $\mathfrak{g}_{2}$ and as the Hochschild-Kostant-Rosenberg map $\psi^{[1]}$ is injective from $\mathfrak{g}_{1}=H\left(\mathfrak{g}_{2}, b=d_{2}^{[1]}\right)$ to $\mathfrak{g}_{2}$, we get

$$
d_{1}^{\prime[2]}=d_{1}^{[2]} .
$$

- Let us prove (3.7): we have $d_{2}^{[1]}(-A)=-\sum_{k=2}^{n} d_{2}^{[1]} d_{2}^{[k]} \psi^{[\leq n-k+1]}$. Using $d_{2} d_{2}=0$, we get

$$
\begin{aligned}
d_{2}^{[1]}(-A) & =\sum_{k=2}^{n}\left(\sum_{l=2}^{k} d_{2}^{[l]} d_{2}^{[k+1-l]}\right) \psi^{[\leq n-k+1]} \\
& =\sum_{l=2}^{n} d_{2}^{[l]}\left(\sum_{k=l}^{n} d_{2}^{[k+1-l]} \psi^{[\leq n-k+1]}\right) .
\end{aligned}
$$

Clearly, we have $\sum_{k=l}^{n} d_{2}^{[k+1-l]} \psi^{[\leq n-k+1]}=\sum_{k=1}^{n-l+1} d_{2}^{[k]} \psi^{[\leq n-k+2-l]}$. Using once again $d_{1}^{[a]} d_{1}^{[b]} \psi^{[c]}=0$ on $V_{1}^{[n]}$ for $a+b+c>n+2$, we add terms $\left(\psi^{\left[n-k+2-l+k^{\prime}\right]}\right)_{0 \leq k^{\prime} \leq k-1}$ to $\psi^{[\leq n-k+2-l]}$ without changing the previous equality. Thus we have

$$
d_{2}^{[1]}(-A)=\sum_{l=2}^{n} d_{2}^{[l]}\left(\sum_{k=1}^{n-l+1} d_{2}^{[k]}\right) \psi^{[\leq n+1-l]}=\sum_{l=2}^{n} d_{2}^{[l]} d_{2}^{[\leq n+1-l]} \psi^{[\leq n+1-l]} .
$$

Since $\left(d_{2}^{[l]}\right)_{l \geq 2}$ map $V_{2}^{[\leq k]}$ into $V_{2}^{[\leq k-1]}$, the previous equality has non-trivial terms only on $V_{1}^{[\leq n-1]}$. Thus we can apply the induction hypothesis $\psi^{[\leq k]} d_{1}^{\prime[\leq k]}=d_{2}^{[\leq k]} \psi^{[\leq k]}$ on $V_{1}^{[\leq k]}$ for $k \leq n-1$. We get

$$
d_{2}^{[1]}(-A)=\sum_{l=2}^{n} d_{2}^{[l]} \psi^{[\leq n+1-l]} d_{1}^{[\leq n+1-l]} .
$$

We have now

$$
d_{2}^{[1]}(B-A)=d_{2}^{[1]} \sum_{k=2}^{n-1} \psi^{[\leq n-k+1]} d_{1}^{\prime^{[k]}}+\sum_{l=2}^{n} d_{2}^{[l]} \psi^{[\leq n+1-l]} d_{1}^{\prime[\leq n+1-l]} .
$$

The term corresponding to $l=n$ vanishes since ${d^{\prime}}_{1}^{[1]}=0$. Using a previous argument on $V_{1}^{[n]}$ for $d_{2}^{[a]} \psi^{[b]} d_{1}^{\prime[c]}$, we add maps $\psi^{\left[p+p^{\prime}\right]}\left(p^{\prime} \geq 0\right)$ to $\psi^{[\leq p]}$. If we then reindex the sum with respect to the terms $d_{1}^{[[l]}$, we get

$$
d_{2}^{[1]}(B-A)=\sum_{l=2}^{n-1} d_{2}^{[\leq n+1-l]} \psi^{[\leq n-1]} d_{1}^{[l]} .
$$


Therefore we have proved that $d_{2}^{[1]}(B-A)=\sum_{l=2}^{n-1} d_{2}^{[\leq n+1-l]} \psi^{[\leq n+1-l]} d_{1}^{[l]}$. Since $d_{1}^{\prime[l]}\left(V_{1}^{[\leq k]}\right) \subset V_{1}^{[\leq k-1]}$, we can again apply the induction hypothesis, thus getting

$$
d_{2}^{[1]}(B-A)=\sum_{l=2}^{n-1} \psi^{[\leq n+1-l]} d_{1}^{[\leq n+1-l]} d_{1}^{\prime[l]}=0
$$

because $d_{1}^{[1]}=0$ and $d_{1}^{\prime[\leq n-1]} d_{1}^{[\leq n-1]}=0$ on $V_{1}^{[\leq n]}$, again by the induction hypothesis. - We will finally prove (3.8) that is to say $d_{1}^{\prime[\leq n]} d_{1}^{\prime[\leq n]}=0$ on $V_{1}^{[\leq n+1]}$. As $\psi^{[1]}$ is a quasi-isomophism between $\left(\mathfrak{g}_{1}, 0\right)$ and $\left(\mathfrak{g}_{2}, b=d_{2}^{[1]}\right)$, this is equivalent to say that $\psi^{[1]} d_{1}^{[\leq n]} d_{1}^{[\leq n]}$ is a boundary on $V_{1}^{[\leq n+1]}$. Using a previous degree argument, we get the following identity on $V_{1}^{[\leq n+1]}$ :

$$
\psi^{[1]} d_{1}^{\prime[\leq n]} d_{1}^{\prime[\leq n]}=\psi^{[\leq n]} d_{1}^{\prime[\leq n]} d_{1}^{\prime[\leq n]} .
$$

By definition of $d_{1}^{\prime[\leq n]}$ we can write $\psi^{[\leq n]} d_{1}^{[\leq n]}=d_{2}^{[\leq n]} \psi^{[\leq n]}$ as $d_{1}^{\prime[\leq n]} \operatorname{maps} V_{1}^{[\leq n+1]}$ to $V_{1}^{[\leq n]}$. Thus it is sufficient to prove that $d_{2}^{[\leq n]} \psi^{[\leq n]} d_{1}^{[\leq n]}$ is a boundary when restricted to $V_{1}^{[\leq n+1]}$.

Now we have

$$
d_{2}^{[\leq n]} \psi^{[\leq n]} d_{1}^{\prime^{[\leq n]}}=b \psi^{[\leq n]} d_{1}^{[\leq n]}+\sum_{2 \leq k \leq n} d_{2}^{[\leq k]} \psi^{[\leq n]} d_{1}^{[\leq n]} .
$$

Since $\sum_{2 \leq k \leq n} d_{2}^{[\leq k]}$ maps $V_{2}^{[\leq k]}$ to $V_{2}^{[\leq k-1]}$, the linear combination of maps

$$
\sum_{2 \leq k \leq n} d_{2}^{[\leq k]} \psi^{[\leq n]} d_{1}^{[\leq n]}
$$

has non-trivial summands only on $V_{1}^{[\leq n+1]}$. On the latter space we have

$$
\sum_{2 \leq k \leq n} d_{2}^{[\leq k]} \psi^{[\leq n]} d_{1}^{\prime[\leq n]}=\sum_{2 \leq k \leq n} d_{2}^{[\leq k]} d_{2}^{[\leq n]} \psi^{[\leq n]},
$$

by definition of $d_{1}^{\prime[\leq n]}$. Hence, the following identities hold on $V_{1}^{[\leq n+1]}$ :

$$
\begin{aligned}
d_{2}^{[\leq n]} \psi^{[\leq n]} d_{1}^{\prime[\leq n]} & =b \psi^{[\leq n]} d_{1}^{[\leq n]}-b d_{2}^{[\leq n]} \psi^{[\leq n]}+d_{2}^{[\leq n]} d_{2}^{[\leq n]} \psi^{[\leq n]} \\
& =b \psi^{[\leq n]} d_{1}^{[\leq n]}-b d_{2}^{[\leq n]} \psi^{[\leq n]}
\end{aligned}
$$

as $d_{2} d_{2}=0$.

Conclusion : The only tool we have used in this section is the existence of a quasiisomorphism between the complexes $\left(\mathfrak{g}_{1}, 0\right)$ and $\left(\mathfrak{g}_{2}, b\right)$. Since we know explicit homotopy formulas for such a quasi-isomorphism (see [DL], [Ha]), we obtain explicit formulas for $d_{1}^{\prime}{ }^{[k]}$ and $\psi^{[k]}$. 


\section{A $G_{\infty}$-morphism $\psi^{\prime}:\left(\Lambda \underline{\mathfrak{g}_{1}^{\otimes}}, d_{1}\right) \rightarrow\left(\Lambda \underline{\mathfrak{g}_{1}^{\otimes}}, d_{1}^{\prime}\right)$}

In this section, we will prove the following proposition.

Proposition 4.1. - If the complex $\left(\operatorname{Hom}\left(\Lambda \underline{\mathfrak{g}_{1}^{\otimes}}, \Lambda \underline{\left.\mathfrak{g}_{1}^{\otimes} \cdot\right)},\left[m_{1}^{1,1}+m_{1}^{2},-\right]\right)\right.$ is acyclic, then there exists a $G_{\infty}$-morphism $\psi^{\prime}:\left(\Lambda \underline{\mathfrak{g}_{1}^{\otimes}}, d_{1}\right) \rightarrow\left(\Lambda \underline{\mathfrak{g}_{1}^{\otimes}}, d_{1}^{\prime}\right)$ such that the induced map $\psi^{[1]}: \mathfrak{g}_{1} \rightarrow \mathfrak{g}_{1}$ is the identity.

We will use the same notations for $V_{1}^{[n]}, V_{1}^{[\leq n]},{d^{\prime}}_{1}^{[n]}$ and ${d^{\prime}}_{1}^{[\leq n]}$ as in Section 3. We also denote

and similarly

$$
d_{1}=\sum_{n \geq 1} d_{1}^{[n]} \quad \text { and } \quad d_{1}^{[\leq n]}=\sum_{1 \leq k \leq n} d_{1}^{[k]}
$$

$$
\psi^{\prime}=\sum_{n \geq 1} \psi^{[n]} \quad \text { and } \quad \psi^{\prime[\leq n]}=\sum_{1 \leq k \leq n} \psi^{[n]} .
$$

Proof: We will build the maps $\psi^{\prime[n]}$ by induction as in Section 3. For $\psi^{\prime^{[1]}}$ we have to set:

$$
\psi^{\prime[1]}=\text { Id (the identity map). }
$$

Suppose we have built maps $\left(\psi^{[[i]}\right)_{i \leq n-1}$ satisfying

$$
\psi^{[\leq n-1]} d_{1}^{[\leq n]}=d_{1}^{\prime[\leq n]} \psi^{\prime[\leq n-1]}
$$

on $V_{1}^{[\leq n]}\left(d_{1}^{\prime}[\leq n]\right.$ maps $V_{1}^{[\leq l]}$ to $\left.V_{1}^{[\leq l-1]}\right)$. Expliciting the equation $\psi^{\prime} d_{1}=d_{1}^{\prime} \psi^{\prime}$ on $V_{1}^{[n+1]}$, we get

$$
\psi^{\prime[\leq n]} d_{1}^{[\leq n+1]}=d_{1}^{\prime[\leq n+1]} \psi^{\prime[\leq n]} .
$$

If we now take into account that $d_{1}^{[i]}=0$ for $i \neq 2, d_{1}^{{ }^{[1]}}=0$ and that on $V_{1}^{[n+1]}$ we have $\psi^{{ }^{[k]}} d_{1}^{[l]}=d_{1}^{\prime}{ }^{[\leq k]} \psi^{[l]}=0$ for $k+l>n+2$, the identity (4.9) becomes

$$
\psi^{\prime[\leq n]} d_{1}^{[2]}=\sum_{k=2}^{n+1} d_{1}^{\prime[k]} \psi^{[\leq n-k+2]} .
$$

We have seen in the previous section that $d_{1}^{\prime}{ }^{[2]}=d_{1}{ }^{[2]}$. Thus (4.9) is equivalent to

$$
d_{1}{ }^{[2]} \psi^{\prime[\leq n]}-\psi^{\prime[\leq n]} d_{1}{ }^{[2]}=\left[d_{1}{ }^{[2]}, \psi^{\prime[\leq n]}\right]=-\sum_{k=3}^{n+1}{d_{1}^{\prime}}^{[k]} \psi^{\prime[\leq n-k+2]} .
$$

Notice that $d_{1}^{[2]}=m_{1}^{1,1}+m_{1}^{2}$. By the acyclicity of the complex $\left(\operatorname{End}\left(\Lambda \cdot \mathfrak{g}_{1}^{\otimes \cdot}\right),\left[d_{1}^{[2]},-\right]\right)$, the construction of $\psi^{[\leq n]}$ will be possible when $\sum_{k=3}^{n+1} d_{1}^{[k]} \psi^{\prime[\leq n-k+2]}$ is a cocycle in this complex. Thus, to finish the proof, we have to check that

$$
\left[d_{1}^{[2]}, \sum_{k=3}^{n+1}{d_{1}^{\prime}}^{[k]} \psi^{\prime[\leq n-k+2]}\right]=0 \text { on } V_{1}^{[n+1]} .
$$


We have

$$
D_{n}=\left[d_{1}^{[2]}, \sum_{k=3}^{n+1} d_{1}^{\prime[k]} \psi^{\prime[\leq n-k+2]}\right]=\left[d_{1}^{[2]}, \sum_{k=1}^{n-1} d_{1}^{\left[{ }^{[n+2-k]}\right.} \psi^{\prime[\leq k]}\right] .
$$

It follows that we can write

$$
-D_{n}=\sum_{k=1}^{n-1}\left[d_{1}^{[2]}, d_{1}^{\prime[n+2-k]}\right] \psi^{\prime[\leq k]}-\sum_{k=1}^{n-1} d_{1}^{\prime[n+2-k]}\left[d_{1}^{[2]}, \psi^{\prime[\leq k]}\right] .
$$

Using the induction hypothesis for $\left(\psi^{\prime[\leq k]}\right)_{k \leq n-1}$, we get

$$
\left[d_{1}{ }^{[2]}, \psi^{\prime[\leq k]}\right]=-\sum_{l=3}^{k+1} d_{1}^{\prime[l]} \psi^{\prime[\leq k-l+2]}=-\sum_{l=1}^{k-1} d_{1}^{[k+2-l]} \psi^{\prime[\leq l]}
$$

on $V_{1}^{[\leq k+1]}$. The equation 4.11 then becomes

$$
-D_{n}=\sum_{k=1}^{n-1}\left[d_{1}^{[2]}, d_{1}^{\prime[n+2-k]}\right] \psi^{\prime[\leq k]}+\sum_{k=1}^{n-1} d_{1}^{\prime[n+2-k]}\left(\sum_{l=1}^{k-1} d_{1}^{\prime[k+2-l]} \psi^{\prime[\leq l]}\right) .
$$

Finally, we have

$$
-D_{n}=\sum_{k=1}^{n-1}\left[d_{1}^{[2]}, d_{1}^{\prime[n+2-k]}\right] \psi^{\prime[\leq k]}+\sum_{l=1}^{n-2}\left(\sum_{k=l+1}^{n-1} d_{1}^{\prime[n+2-k]} d_{1}^{\prime[k+2-l]}\right) \psi^{\prime[\leq l]} .
$$

This implies

$$
-D_{n}=\sum_{k=1}^{n-1}\left(\left[d_{1}^{\prime[2]}, d_{1}^{\prime[n+2-k]}\right]+\sum_{p=k+1}^{n-1} d_{1}^{{ }^{[n+2-p]}} d_{1}^{\prime[p+2-k]}\right) \psi^{\prime[\leq k]} .
$$

But the maps

$$
\left[d_{1}^{\prime[2]}, d_{1}^{\prime}{ }^{[n+2-k]}\right]+\sum_{p=k+1}^{n-1} d_{1}^{{ }^{[n+2-p]}} d_{1}^{\prime[p+2-k]}=\sum_{q=2}^{n+2-k} d_{1}^{\prime[q]} d_{1}^{{ }^{[n+4-q-k]}}
$$

are zero because $d_{1}^{\prime} d_{1}^{\prime}=0$ on $V_{1}^{[\leq n+2-k]}$. This yields the result.

5. Acyclicity of the complex $\left(\operatorname{Hom}\left(\Lambda \underline{\mathfrak{g}_{1}^{\otimes}}, \Lambda \underline{\mathfrak{g}_{1}^{\otimes \cdot}}\right),\left[m_{1}^{1,1}+m_{1}^{2},-\right]\right)$

In this section the manifold $M$ is supposed to be the Euclidian space $\mathbb{R}^{m}$ for $m \geq 1$. We prove the following proposition:

Proposition 5.1. - If $M=\mathbb{R}^{m}$, the cochain complex $\left(\operatorname{End}\left(\Lambda \underline{\mathfrak{g}}_{1}^{\otimes \cdot}\right),\left[m_{1}^{1,1}+m_{1}^{2},-\right]\right)$ is acyclic. 
Proof: Since coalgebras maps $\Lambda \mathfrak{g}_{1}^{\otimes \cdot} \rightarrow \Lambda \mathfrak{g}_{1}^{\otimes \cdot}$ are in one to one correspondence with maps $\Lambda \mathfrak{g}_{1}^{\otimes \cdot} \rightarrow \mathfrak{g}_{1}$, we are left to check that the cochain complex

$$
\left(\operatorname{Hom}\left(\Lambda \underline{\mathfrak{g}_{1}^{\otimes}}, \mathfrak{g}_{1}\right),\left[m_{1}^{1,1}+m_{1}^{2},-\right]\right)
$$

is acyclic.

First we introduce an "external" bigrading on the cochain complex induced by duality from the following bigrading on $\Lambda \cdot \underline{\mathfrak{g}_{1}}$ :

$$
|x|^{\mathrm{e}}=\left(p_{1}-1+\cdots+p_{n}-1, n-1\right)
$$

if $x \in \underline{\mathfrak{g}_{1}^{\otimes p_{1}}} \wedge \cdots \wedge \underline{\mathfrak{g}_{1}^{\otimes p_{n}}}$. Let the internal degree of $x \in \mathfrak{g}_{1}$ be $|x|^{\mathbf{i}}=|x|+1$, where $|x|$ is the usual degree of an element of $\mathfrak{g}_{1}$. One recovers the usual degree on $\Lambda \mathfrak{g}_{1}^{\otimes \cdot}$ by

$$
|x|=|x|_{\text {tot }}^{\mathrm{e}}+\sum_{i, k}\left|x_{i}^{k}\right|^{\mathrm{i}}
$$

where $|x|_{\text {tot }}^{\mathrm{e}}$ is the sum of the two components of $|x|^{\mathrm{e}}$.

The exterior product $m_{1}^{2}$ makes $\mathfrak{g}_{1}$ into an associative algebra which is graded commutative for the inner degree. For any vector space $V$, the space $\mathfrak{g}_{1} \otimes V$ is a $\mathfrak{g}_{1}$-module equiped with a $\mathfrak{g}_{1}$-action by multiplication on the first factor. Observe that

$$
\begin{aligned}
& \left(\operatorname{Hom}\left(\Lambda \underline{\mathfrak{g}_{1}^{\otimes \cdot}}, \mathfrak{g}_{1}\right),\left[m_{1}^{1,1}+m_{1}^{2},-\right]\right) \cong\left(\operatorname{Hom}_{\mathfrak{g}_{1}}\left(\mathfrak{g}_{1} \otimes \Lambda \underline{\mathfrak{g}_{1}^{\otimes \cdot}}, \mathfrak{g}_{1}\right),\left[m_{1}^{1,1}+m_{1}^{2},-\right]\right), \\
& \cong\left(\operatorname{Hom}_{\mathfrak{g}_{1}}\left(\Lambda_{\mathfrak{g}_{1}} \mathfrak{g}_{1} \otimes \underline{\mathfrak{g}_{1}^{\otimes}}, \mathfrak{g}_{1}\right),\left[m_{1}^{1,1}+m_{1}^{2},-\right]\right)
\end{aligned}
$$

where $\mathfrak{g}_{1}$ acts (on the right and on the left) on itself by the multiplication $m_{1}^{2}$.

We now prove acyclicity of this last cochain complex. The codifferential $(\delta)^{*}=$ $\left[m_{1}^{1,1}+m_{1}^{2},-\right]$ splits in two parts $\left(\delta_{1}^{2}\right)^{*}+\left(\delta_{1}^{1,1}\right)^{*}=(\delta)^{*}$ where $\left(\delta_{1}^{2}\right)^{*}$ is the codifferential of bidegree $(1,0)$ induced by $m_{1}^{2}$ and $\left(\delta_{1}^{1,1}\right)^{*}$ is the one of bidegree $(0,1)$ induced by $m_{1}^{1,1}$. Thus, $\operatorname{Hom}_{\mathfrak{g}_{1}}\left(\Lambda_{\mathfrak{g}_{1}} \mathfrak{g}_{1} \otimes \underline{\mathfrak{g}_{1}^{\otimes \cdot}}, \mathfrak{g}_{1}\right)$ endowed with the bigrading $|-|^{\mathrm{e}}$ is a bicomplex lying in the first quadrant. The bigrading of an element $x \in \mathfrak{g}_{1} \otimes \mathfrak{g}_{1}^{\otimes p_{1}} \wedge \ldots \wedge \mathfrak{g}_{1} \otimes \mathfrak{g}_{1}^{\otimes p_{n}}$ is $|x|^{\mathrm{e}}=\left(p_{1}-1+\ldots+p_{n}-1, n-1\right)$.

The codifferential $\left(\delta_{1}^{2}\right)^{*}$ is dual to a differential $\delta_{1}^{2}$. It is a standard calculation (see [Lo1] 1.5 for example) to show that $\delta_{1}^{2}$, restricted to each summand $\mathfrak{g}_{1} \otimes \mathfrak{g}_{1}^{\otimes \cdot}$, is the usual Harrison boundary, that is to say the image of the Hochschild differential $d$ acting on $\mathfrak{g}_{1}^{\otimes \cdot+1}$ onto its quotient $\mathfrak{g}_{1} \otimes \mathfrak{g}_{1}^{\otimes \cdot}$ by the shuffles.

We now use the fact that $\left(\mathfrak{g}_{1}, m_{1}^{2}\right)=(\Gamma(M, \Lambda T M), \wedge)$ is a polynomial algebra. Denote by $\Omega_{\mathfrak{g}_{1}}$ the module of Kähler differential one-forms of the algebra $\mathfrak{g}_{1}$. Let $J: \mathfrak{g}_{1}^{\otimes \cdot+1} \rightarrow \Lambda \Omega_{\mathfrak{g}_{1}}$ be the map which sends $x_{0} \otimes \cdots \otimes x_{n}$ to $x_{0} d x_{1} \cdots d x_{n}$ and $I: \Lambda \cdot \Omega_{\mathfrak{g}_{1}} \rightarrow \mathfrak{g}_{1}^{\otimes \cdot+1}$ be the anti-symmetrization map given by

$$
J\left(x_{0} d x_{1} \cdots d x_{n}\right)=\sum_{\sigma \in S_{n}} \frac{(-1)^{\sigma}}{n !} \varepsilon(\sigma) x_{0} \otimes x_{\sigma^{-1}(1)} \cdots \otimes x_{\sigma^{-1}(n)}
$$

where $S_{n}$ is the permutation group of $\{1, \cdots, n\},(-1)^{\sigma}$ is the sign of $\sigma$ and $\varepsilon(\sigma)$ the Koszul-Quillen sign (see Section 1). It is easy to check that $J \circ I=\mathrm{Id}$. 
It is known from [Ha] that there exists a homotopy $s: \mathfrak{g}_{1}^{\otimes \cdot+1} \rightarrow \mathfrak{g}_{1}^{\otimes \cdot+2}$ such that $I \circ J=\operatorname{Id}+d \circ s+s \circ d$. We denote $P$ the natural projection $\mathfrak{g}_{1}^{\otimes \cdot+1} \rightarrow \mathfrak{g}_{1} \otimes \mathfrak{g}_{1}^{\otimes}$. It is a standard computation (see [Lo2]) that the map $J$ factors through $\mathfrak{g}_{1} \otimes \underline{\mathfrak{g}_{1}^{\otimes \cdot}}$ to give a map $J^{\prime}: \mathfrak{g}_{1} \otimes \underline{\mathfrak{g}_{1}^{\otimes}} \rightarrow \Omega_{\mathfrak{g}_{1}}$. There is also a map $I^{\prime}=P \circ J: \Omega_{\mathfrak{g}_{1}} \rightarrow \mathfrak{g}_{1} \otimes \underline{\mathfrak{g}_{1}^{\otimes \cdot}}$. Clearly $J^{\prime} \circ I^{\prime}=$ Id. Since the map $P$ commutes with the differential $d$, the map $s^{\prime}=P \circ s$ satisfies

$$
I^{\prime} \circ J^{\prime}=\mathrm{Id}+d \circ s^{\prime}+s^{\prime} \circ d
$$

The map $s^{\prime}$ extends uniquely into a degree 1 homotopy $h$ to $\Lambda_{\mathfrak{g}_{1}}^{\cdot} \mathfrak{g}_{1} \otimes \mathfrak{g}_{1}^{\otimes}$. so that $\Lambda I^{\prime} \circ \Lambda J^{\prime}=\operatorname{Id}+\delta_{1}^{2} \circ h+h \circ \delta_{1}^{2}$, where $\Lambda I^{\prime}, \Lambda J^{\prime}$ are the extensions of the degree zero maps $I^{\prime}$ and $J^{\prime}$. Moreover $\Lambda J^{\prime} \circ \Lambda I^{\prime}=\operatorname{Id}$ and $\Lambda_{\mathfrak{g}_{1}} \Omega_{\mathfrak{g}_{1}}$ is a (special) deformation retract of $\Lambda_{\mathfrak{g}_{1}} \mathfrak{g}_{1} \otimes \mathfrak{g}_{1}^{\otimes \cdot}($ see $[\mathbf{H a}])$. We denote $p: \Lambda_{\mathfrak{g}_{1}} \mathfrak{g}_{1} \otimes \mathfrak{g}_{1}^{\otimes} \rightarrow \Lambda_{g_{1}} \Omega_{g_{1}}$ the projection. Since $\Lambda_{\mathfrak{g}_{1}} \mathfrak{g}_{1} \otimes \mathfrak{g}_{1}^{\otimes} \cdot \overline{\text { is }}$ a bicomplex with differential $\delta=\delta_{1,1}^{2}+\delta_{1}^{1,1}$, it follows from $[\mathbf{K a}]$, Section 3 that there exists a map $u: \Lambda_{g_{1}} \Omega_{g_{1}} \rightarrow \Lambda_{\mathfrak{g}_{1}}^{\cdot} \mathfrak{g}_{1} \otimes \underline{\mathfrak{g}_{1}} \otimes$ and a (degree one) map $H: \Lambda_{\mathfrak{g}_{1}}^{\cdot} \mathfrak{g}_{1} \otimes \mathfrak{g}_{1}^{\otimes \cdot} \rightarrow \Lambda_{\mathfrak{g}_{1}}^{\cdot} \mathfrak{g}_{1} \otimes \mathfrak{g}_{1}^{\otimes \cdot}[1]$ such that $p u=\operatorname{Id}$ and $u p=\operatorname{Id}+\delta H+$ $H \delta$. Hence the cohomology we are looking for is the cohomology of the complex $\left(\operatorname{Hom}_{\mathfrak{g}_{1}}\left(\Lambda_{\mathfrak{g}_{1}} \Omega_{g_{1}}, \mathfrak{g}_{1}\right), \delta_{1}^{1,1}\right)$ which sits in the complex

$$
\left(\operatorname{Hom}_{\mathfrak{g}_{1}}\left(\Lambda_{\mathfrak{g}_{1}}^{\cdot} g_{1}, \mathfrak{g}_{1}\right), \delta_{1}^{1,1}\right) \cong\left(\operatorname{Hom}_{\mathfrak{g}_{1}}\left(\mathfrak{g}_{1} \otimes \Lambda \mathfrak{g}_{1}, \mathfrak{g}_{1}\right), \delta_{1}^{1,1}\right)
$$

In particular, the differential $\delta_{1}^{1,1}$ is induced by the usual exterior derivative (see $[\mathbf{H K R}])$ on $\operatorname{Hom}_{\mathfrak{g}_{1}}\left(\mathfrak{g}_{1} \otimes \Lambda \mathfrak{g}_{1}^{\otimes \cdot}, \mathfrak{g}_{1}\right)$. To finish the proof, we proceed as in [Ta] and [Hi] Recall from the introduction that $A=C^{\infty}\left(\mathbb{R}^{m}\right)$ is the algebra of smooth functions on $\mathbb{R}^{m}$. Let $\operatorname{Der}(A)=\Omega_{A}^{*}$ be the space of smooth derivations on $A$. Since $\mathfrak{g}_{1}$ is a $A$-module, by transitivity of the space of Kähler differentials for smooth manifolds, one has

$$
\Omega_{\mathfrak{g}_{1}} \cong \mathfrak{g}_{1} \otimes \Omega_{A} \oplus \Omega_{\mathfrak{g}_{1} / A}
$$

Since $\mathfrak{g}_{1} \cong \Lambda_{A}^{*} \operatorname{Der}(A)$, we find that $\Omega_{\mathfrak{g}_{1} / A} \cong \mathfrak{g}_{1} \otimes \operatorname{Der}(A)$ (with grading shifted by minus one on $\operatorname{Der}(A)$ ). Hence (see [Ta].3.5) there is an isomorphism

$$
\left(\operatorname{Hom}_{\mathfrak{g}_{1}}\left(\Lambda_{\mathfrak{g}_{1}}^{\cdot} \Omega_{g_{1}}, \mathfrak{g}_{1}\right), \delta_{1}^{1,1}\right) \cong\left(\Lambda^{1+\cdot} \Omega_{\mathfrak{g}_{1}}, d_{d R}\right)
$$

where $d_{d R}$ is de Rham's differential (the degree on the left hand of the isomorphism is the one induced by the inner degree of $\left.\mathfrak{g}_{1}\right)$. When $\mathfrak{g}_{1}=\Gamma\left(\mathbb{R}^{n}, \Lambda \mathbb{R}^{n}\right)$ this complex is acyclic.

Remark: At every step of this proof, it is possible to construct explicit homotopy formulas. So the coefficients $\psi^{[}[n]$ built in this section can be expressed in an explicit way from the $G_{\infty}$-structure on $\mathfrak{g}_{2}$.

Corollary 5.2. - If $\mathfrak{g}_{1}=\Gamma\left(\mathbb{R}^{m}, \Lambda T \mathbb{R}^{m}\right)$, then there exists a $G_{\infty}$-morphism $\psi^{\prime}$ : $\left(\Lambda \underline{\mathfrak{g}}_{1}^{\otimes \cdot}, d_{1}\right) \rightarrow\left(\Lambda \underline{\mathfrak{g}}_{1}^{\otimes \cdot}, d_{1}^{\prime}\right)$ such that the induced map $\psi^{[1]}: \mathfrak{g}_{1} \rightarrow \mathfrak{g}_{1}$ is the identity.

Proof: It is an immediate consequence of Propositions 4.1 and 5.1. 


\section{Consequences when $M$ is a Poisson manifold}

From the Sections 3, 4 and 5 we know that the map

$$
\phi=\psi \circ \psi^{\prime}:\left(\Lambda \underline{\mathfrak{g}_{1}^{\otimes}}, d_{1}\right) \rightarrow\left(\Lambda \underline{\mathfrak{g}_{2}^{\otimes}}, d_{2}\right)
$$

is a $G_{\infty}$-morphism when $M=\mathbb{R}^{m}$; in other words, we have the identity

$$
\phi \circ d_{1}=d_{2} \circ \phi \text { on } \Lambda \underline{\mathfrak{g}_{1}^{\otimes}} .
$$

Since $\phi: \Lambda \mathfrak{g}_{1}^{\otimes \cdot} \rightarrow \Lambda \mathfrak{g}_{2}^{\otimes \cdot}$ is a coalgebra map, it restricts to the subcoalgebra $\Lambda \mathfrak{g}_{1}$ to give a coalgebra map $\overline{\Lambda^{\prime}} \mathfrak{g}_{1} \rightarrow \Lambda^{\prime} \mathfrak{g}_{2}$. The restriction of $d_{1}$ and $d_{2}$ are respectively the codifferential induced by $m_{1}^{1,1}$ and the codifferential induced by $b+m_{2}^{1,1}$. When we restrict these maps to $\Lambda^{\prime} \mathfrak{g}_{1}$ and $\Lambda^{\prime} \mathfrak{g}_{2}$, the previous equality (6.12) still holds with the difference that, now, $d_{1}$ and $d_{2}$ are the differential defining the $L_{\infty}$-structures on $\mathfrak{g}_{1}$ and $\mathfrak{g}_{2}$ of Section 1 . So the restriction of $\phi$ to these coalgebras yields a morphism of differential coalgebras

$$
\phi:\left(\Lambda \mathfrak{g}_{1}, d_{1}\right) \rightarrow\left(\Lambda \mathfrak{g}_{2}, d_{2}\right) .
$$

Thus we have constructed the desired $L_{\infty}$-morphism between $\left(\mathfrak{g}_{1}, d_{1}\right)$ and $\left(g_{2}, d_{2}\right)$.

Remark: Similarly to Definitions $1.1,1.3$, one can define, on a vector space $\mathfrak{g}$, a $C_{\infty}$-algebra structure given by degree one maps $a^{m}: \mathfrak{g}^{\otimes n} \rightarrow \mathfrak{g}$ such that if we extend them to maps $\oplus \underline{\mathfrak{g}}^{\otimes \cdot} \rightarrow \oplus \underline{\mathfrak{g}}^{\otimes \cdot}$, then $D=\sum a^{m}$ satifies $D \circ D=0$. In particular, $a^{2}$ yields a commutative operation on $\mathfrak{g}, a^{1}$ a differential and the product $a^{2}$ is associative up to homotopies for the differential $a^{1}$. Let us then consider the free Lie coalgebras $\oplus_{n \geq 0} \underline{\mathfrak{g}}_{1}{ }^{\otimes n}$ and $\oplus_{n \geq 0} \underline{\mathfrak{g}}_{2}{ }^{\otimes n}$. They are also subcoalgebras of respectively $\Lambda \underline{\mathfrak{g}}_{1}^{\otimes \cdot}$ and $\Lambda \underline{\mathfrak{g}}_{2}^{\otimes}$. Hence we can restrict $\phi$ into a coalgebra map $\phi: \oplus \underline{\mathfrak{g}}^{\otimes n} \rightarrow \oplus \underline{\mathfrak{g}}^{\otimes n}$. Denoting $\widehat{D_{1}=} m_{1}^{2}$ the codifferential induced by the exterior product $\wedge$, and $\overline{D_{2}}$ the codifferential induced by $\sum_{n \geq 0} m_{2}^{n}$, the map $\phi$ yields a differential coalgebra morphism

$$
\phi:\left(\oplus \underline{\mathfrak{g}}^{\otimes n}, D_{1}\right) \rightarrow\left(\oplus \underline{\mathfrak{g}}^{\otimes n}, D_{2}\right),
$$

hence, a morphism of $C_{\infty}$-algebras between $\left(\mathfrak{g}_{1}, D_{1}\right)$ and $\left(\mathfrak{g}_{2}, D_{2}\right)$. Through the Etingof-Kazdhan equivalence used in Proposition 2.2, this implies that there is a morphism of $A_{\infty}$-algebras between $\left(\mathfrak{g}_{1}, \wedge\right)$ and $\left(\mathfrak{g}_{2}, \cup\right)$. More precisely, it means that there is a morphism $\left(\oplus \mathfrak{g}_{1}^{\otimes n}, \wedge\right) \rightarrow\left(\oplus \mathfrak{g}_{2}^{\otimes n}, b+\cup\right)$ of differential coalgebras between the tensor coalgebras of $\mathfrak{g}_{1}$ and $\mathfrak{g}_{2}$. Details on $C_{\infty}$ and $A_{\infty}$-structures can be found in $[\mathbf{G K}]$ and $[\mathbf{S t}]$.

From now on, we will suppose that the manifold $M$ is a Poisson manifold equipped with a Poisson tensor $\pi$ (satisfying $[\pi, \pi]_{S}=0$ ). The $L_{\infty}$-map $\phi$ allows us to construct a star-product on $M$ (see [BFFLS1]). If $\hbar$ is a formal parameter and if we impose $\phi$ to be $\mathbb{R}[[\hbar]]$-linear, $\phi$ extends to a $L_{\infty}$-morphism between $\mathfrak{g}_{1}[[h]]$ and $\mathfrak{g}_{2}[[h]]$. Set $\Pi_{\hbar}=\sum_{n \geq 0} \hbar^{n} \Lambda^{n} \pi \in \Lambda \cdot \mathfrak{g}_{1}$, where $\Lambda^{n} \pi=\underbrace{\pi \wedge \ldots \wedge \pi}_{n \text { times }}$. If we define $m_{\star}=\phi\left(\Pi_{\hbar}\right)$, we get

$$
\left[m_{\star}, m_{\star}\right]_{G}=0 \text {. }
$$


This is a consequence of definition (0.1) of a $L_{\infty}$-morphism given in Section 0 and of the fact that $[\pi, \pi]_{S}=0$ implies $m_{1}^{1,1}\left(\Pi_{\hbar}\right)=0$. The map $m_{\star}$ being an element of $\mathfrak{g}_{2}[[\hbar]]$ of degree one, it defines a bilinear map in $C^{2}(A, A)[[\hbar]]$, where $C^{k}(A, A)[[\hbar]]$ denotes the set of $k-\mathbb{R}[[\hbar]]$-linear maps in $C^{k}(A, A)$. The identity 6.13 implies that $m_{\star}$ is an associative product on $A[[\hbar]]$. Finally, by definition of $\phi$, we have:

$$
m_{\star}=m+\hbar \phi^{1}(\pi)+\sum_{n \geq 2} \hbar^{n} \phi^{n}(\pi, \ldots, \pi)
$$

where $\phi^{1}(\pi)=\{\cdot, \cdot\}$ is the Poisson bracket. This proves that $m_{\star}$ is a star-product on $(M, \pi)$.

The spaces $\mathfrak{g}_{1}$ and $\mathfrak{g}_{2}$ can now be endowed with two new structures: the space $\left(\mathfrak{g}_{1},[-,-]_{S},[\pi,-]_{S}\right)$ becomes a graded differential Lie algebra (and even a Gerstenhaber algebra) whereas $\left(\mathfrak{g}_{2},[-,-]_{G}, b_{\star}\right)$, where $b_{\star}$ is the Hochschild differential corresponding to the deformed product $m_{\star}$, is a new graded differential Lie algebra. As in the case when $\pi=0$, we have the following result $\grave{a}$ la Hochschild-Kostant-Rosenberg.

Theorem 6.1. - The complexes $\left(\mathfrak{g}_{2}[[\hbar]], b_{\star}\right)$ and $\left(\mathfrak{g}_{1}[[\hbar]],[\hbar \pi,-]_{S}\right)$ are quasi-isomorphic.

Proof: Let us denote $\phi_{\hbar}^{1}$ the $\mathbb{R}[[\hbar]]$-linear map $\mathfrak{g}_{1}[[\hbar]] \rightarrow \mathfrak{g}_{2}[[\hbar]]$ given by

$$
\alpha \mapsto \phi_{\hbar}^{1}(\alpha)=\sum_{n \geq 0} \hbar^{n} \phi^{n+1}\left(\Lambda^{n} \pi \wedge \alpha\right)=\phi_{\mathfrak{g}_{1}}\left(\sum_{n \geq 0} \hbar^{n} \Lambda^{n} \pi \wedge \alpha\right)
$$

for $\alpha \in \mathfrak{g}_{1}$, where $\phi_{\mathfrak{g}_{1}}$ denotes the projection of $\phi$ on $\mathfrak{g}_{1}$. Similarly, we write $\phi_{\mathfrak{g}_{1} \wedge \mathfrak{g}_{1}}$ for the projection of $\phi$ on $\mathfrak{g}_{1} \wedge \mathfrak{g}_{1}$. We get

$$
\begin{aligned}
\phi_{\hbar}^{1}\left([\hbar \pi, \alpha]_{S}\right) & =\phi_{\mathfrak{g}_{1}}\left(\sum_{n \geq 0} \hbar^{n} \Lambda^{n} \pi \wedge[\hbar \pi, \alpha]_{S}\right) \\
& =\phi_{\mathfrak{g}_{1}}\left(\sum_{n \geq 0} \hbar^{n+1} m_{1}^{1,1}\left(\Lambda^{n+1} \pi \wedge \alpha\right)\right) \\
& =m_{2}^{1,1}\left(\phi_{\mathfrak{g}_{1} \wedge \mathfrak{g}_{1}}\left(\sum_{n \geq 0} \hbar^{n+1} \Lambda^{n+1} \pi \wedge \alpha\right)\right) \\
& =\left[\phi\left(\sum_{n \geq 0} \hbar^{n} \Lambda^{n} \pi\right), \phi\left(\sum_{n \geq 0} \hbar^{n} \Lambda^{n} \pi \wedge \alpha\right)\right]_{G} \\
& =\left[m_{\star}, \phi_{\hbar}^{1}(\alpha)\right]_{G} \\
& =b_{\star} \phi_{\hbar}^{1}(\alpha) .
\end{aligned}
$$

Thus $\phi_{\hbar}^{1}$ is a morphism of complexes between $\left(\mathfrak{g}_{1},[\hbar \pi,-]_{S}\right)$ and $\left(\mathfrak{g}_{2}[[\hbar]], b_{\star}\right)$. By definition, we can write $\phi_{\hbar}^{1}(\alpha)=\phi^{1}(\alpha)+\sum_{n \geq 1} \hbar^{n} \phi_{\hbar}^{1, n}$ where $\phi_{\hbar}^{1, n}$ are $\mathbb{R}[[\hbar]]$-linear maps. The proof of the theorem is then a consequence of the following lemma. 
Lemma 6.2. - Let $\varphi:(B, 0) \rightarrow(D, d)$ be a quasi-isomorphism of cochain complexes. Suppose we have two deformed complexes $\left(B[[\hbar]], b_{\hbar}\right)$ and $\left(D[[\hbar]], d_{\hbar}\right)$ with $b_{\hbar}=\sum_{n \geq 1} \hbar^{n} b_{n}$ and $d_{\hbar}=d+\sum_{n \geq 1} \hbar^{n} d_{n}$, where $b_{i}$ and $d_{i}$ are $\mathbb{R}[[\hbar]]$-linear maps. Suppose in addition that there exists a morphism of complexes $\varphi_{\hbar}=\varphi+\sum_{n \geq 1} \varphi_{n}$ between $\left(B[[\hbar]], b_{\hbar}\right)$ and $\left(D[[\hbar]], d_{\hbar}\right)$, where $\varphi_{i}$ are $\mathbb{R}[[\hbar]]$-linear maps. Then $\varphi_{\hbar}$ is a quasi-isomorphism.

Proof: Suppose $\delta_{\hbar}=\sum_{n \geq 0} \hbar^{n} \delta_{n} \in D[[\hbar]]$ satisfies $d_{\hbar} \delta_{\hbar}=0$. We will construct $\beta_{n} \in B$ by induction such that $\beta_{\hbar}=\sum_{n \geq 0} \beta_{n}$ satifies $b_{\hbar} \beta_{\hbar}=0$ and $\varphi_{\hbar}\left(\beta_{\hbar}\right)=\delta_{\hbar}$. Since $d_{\hbar} \delta_{\hbar}=0$, we have $d_{0} \delta_{0}=0$, so that $\bar{\delta}_{0}=\varphi_{0}\left(\beta_{0}\right),\left(\beta_{0} \in B\right.$ with $\left.b_{0} \beta_{0}=0\right)$. This follows from $\varphi_{0}$ being a quasi-isomorphism between $(B, 0)$ and $\left(D, d_{0}\right)$.

Suppose we have built $\beta_{0}, \ldots, \beta_{n} \in B$ such that for all $k \leq n$,

$$
\delta_{k}=\sum_{i=0}^{k} \varphi_{i}\left(\beta_{k-i}\right)
$$

and

$$
\sum_{i=0}^{k} b_{i} \beta_{k-i}=0
$$

We have shown that Relations (6.14) and (6.15) hold for $k=0$. We will now construct $\beta_{n+1}$ such that they hold for $k=n+1$. We can reformulate Relation (6.14) as follows:

$$
\varphi_{0}\left(\beta_{n+1}\right)=\delta_{n+1}-\sum_{i=1}^{n+1} \varphi_{i}\left(\beta_{n+1-i}\right) .
$$

Since $\varphi_{0}$ is a quasi-isomorphism between $(B, 0)$ and $\left(D, d_{0}\right)$, this is equivalent to say:

$$
d_{0}\left(\delta_{n+1}\right)-\sum_{i=1}^{n+1} d_{0} \varphi_{i}\left(\beta_{n+1-i}\right)=0 .
$$

Since $d_{\hbar} \delta_{\hbar}=0$ we have $d_{0} \delta_{n+1}=-\sum_{i=1}^{n+1} d_{i} \delta_{n+1-i}$. Therefore

$$
\begin{aligned}
(6.16) & \Longleftrightarrow \sum_{i=1}^{n+1} d_{i} \delta_{n+1-i}+\sum_{i=1}^{n+1} d_{0} \varphi_{i} \beta_{n+1-i}=0 \\
& \Longleftrightarrow \sum_{i=1}^{n+1} d_{i} \sum_{j=0}^{n+1-i} \varphi_{j}\left(\beta_{n+1-i-j}\right)+\sum_{i=1}^{n+1} d_{0} \varphi_{i} \beta_{n+1-i}=0 \\
& \Longleftrightarrow \sum_{i=0}^{n+1} \sum_{j=0}^{n+1-i} d_{i} \varphi_{j}\left(\beta_{n+1-j-i}\right)=0
\end{aligned}
$$

$\left(\beta_{n+1}=0\right.$ by convention). Since $\varphi_{\hbar}$ is a morphism of complexes, we obtain

$$
\sum_{i=0}^{n+1} \sum_{j=0}^{n+1-i} d_{i} \varphi_{j}\left(\beta_{n+1-j-i}\right)=\sum_{i=0}^{n+1} \sum_{j=0}^{n+1-i} \varphi_{i}\left(b_{j} \beta_{n+1-j-i}\right)=\varphi_{0}\left(\sum_{j=0}^{n+1} b_{j} \beta_{n+1-j}\right) \text {. }
$$


So (6.16) $\varphi_{0}\left(\sum_{j=0}^{n+1} b_{j} \beta_{n+1-j}\right)=0$. This relation will be satisfied provided we have proved Relation (6.15) for $k=n+1$. As $\varphi_{0}$ is a quasi-isomorphism of complexes between $(B, 0)$ and $\left(D, d_{0}\right)$ we only have to prove that $\varphi_{0}\left(\sum_{j=1}^{n+1} b_{j} \beta_{n+1-j}\right)$ is a boundary. Using Relarion (6.17), we have

$$
\begin{aligned}
\varphi_{0}\left(\sum_{j=0}^{n+1} b_{j} \beta_{n+1-j}\right) & =\sum_{i=0}^{n+1} \sum_{j=0}^{n+1-i} d_{i} \varphi_{j}\left(\beta_{n+1-j-i}\right) \\
& =d_{0} \sum_{j=0}^{n+1} \varphi_{j}\left(\beta_{n+1-j}\right)+\sum_{i=1}^{n+1} \sum_{j=0}^{n+1-i} d_{i} \varphi_{j}\left(\beta_{n+1-j-i}\right) \\
& =d_{0} \sum_{j=0}^{n+1} \varphi_{j}\left(\beta_{n+1-j}\right)+\sum_{i=1}^{n+1} d_{i} \delta_{n+1-i} \quad \text { (thanks to (6.14)) } \\
& =d_{0} \sum_{j=0}^{n+1} \varphi_{j}\left(\beta_{n+1-j}\right)-d_{0} \delta_{n+1}
\end{aligned}
$$

because $d_{\hbar} \beta_{\hbar}=0$.

It is clear from the previous proof that we can build explicit homotopy formulas for the map $\phi_{\hbar}^{1}$ since in the proof of Theorem 6.1 , we had $\phi_{\hbar}^{1}=\phi^{1}+O(\hbar)$, with $\phi^{1}$ the Hochschild-Kostant-Rosenberg map.

Remark 6.3: Lemma 6.2 also holds for two cochain complexes $(B, b)$ and $(D, d)$, where $b_{\hbar}=b+\sum_{n \geq 1} \hbar^{n} b_{n}$ with $b \neq 0$, but we have no explicit homotopy formulas.

The cochains complexes $\left(B[[\hbar]], b_{\hbar}\right)$ and $\left(D[[\hbar]], d_{\hbar}\right)$ are filtered by the powers of $\hbar$. The $p$-component of the filtration is

$$
F^{p}(B[[\hbar]])=\hbar^{p} B[[\hbar]] \subset B[[\hbar]]
$$

and similarly for $D[[\hbar]]$. The filtrations are decreasing and the differentials $b_{\hbar}$ and $d_{\hbar}$ respects the filtrations as well as the morphism $\varphi_{\hbar}$. Therefore, there are two spectral sequences with first terms given by

$$
E B_{1}^{p, q}=H^{p+q}\left(F^{q} B[[\hbar]] / F^{q+1} B[[\hbar]]\right)
$$

and

$$
E D_{1}^{p, q}=H^{p+q}\left(F^{q} D[[\hbar]] / F^{q+1} D[[\hbar]]\right)
$$

converging respectively to $\left.H^{\cdot}\left(B[[\hbar]], b_{\hbar}\right)\right)$ and $H^{\cdot}\left(D[[h]], d_{\hbar}\right)$. The morphism $\varphi_{\hbar}$ induces a map of spectral sequences $\varphi_{1}^{\hbar}: E B_{1}^{\prime \prime} \rightarrow E D_{1}^{\prime \prime}$.

It is easy to check that $E B_{1}^{p, q} \cong H^{p+q}(B) \hbar^{q}, E D_{1}^{p, q} \cong H^{p+q}(D) \hbar^{q}$ and that the map $\varphi_{1}^{\hbar}$ is induced by the quasi-isomorphim $\varphi$, hence is an isomorphism for all $p, q$. As the spectral sequences are strongly convergent in the sense of $[\mathbf{C E}]$, Section 15.2, it follows that $\varphi_{\hbar}$ induces an isomorphism $\left.H^{\cdot}\left(B[[\hbar]], b_{\hbar}\right)\right) \cong H^{\cdot}\left(D[[h]], d_{\hbar}\right)$.

Now, we are in the situation of Section 1: we have two graded differential Lie algebras $\left(\mathfrak{g}_{1},[-,-]_{S},[\pi,-]_{S}\right)$ and $\left(\mathfrak{g}_{2},[-,-]_{G}, b_{\star}\right)$ such that $H\left(\mathfrak{g}_{2}, b_{\star}\right) \cong H\left(\mathfrak{g}_{1},[\pi,-]_{S}\right)$. 
The quasi-isomorphism $\phi_{\hbar}^{1}$ is not a Lie algebra morphism. The aim of the next section is to construct a $L_{\infty}$-morphism between $\left(\mathfrak{g}_{1},[\pi,-]_{S}\right)$ and $\left(\mathfrak{g}_{2}, b_{\star}\right)$.

\section{A formality theorem for a Poisson manifold}

In this section we define $d_{1 \star}$ the map $d_{1 \star}=m_{1}^{1,1}+m_{1}^{2}+\hbar[\pi,-]_{S}: \Lambda \underline{\mathfrak{g}_{1 \star}^{\otimes}} \rightarrow \Lambda \underline{\mathfrak{g}}_{1 \star}^{\otimes}$, where $\mathfrak{g}_{1 \star}=\mathfrak{g}_{1}[[\hbar]]$. As for the case $\pi=0$, we will construct a $G_{\infty}$-structure on $\mathfrak{g}_{2 \star}=$ $\mathfrak{g}_{2}[[\hbar]]$ given by a differential $d_{2 \star}: \Lambda \underline{\mathfrak{g}}_{2 \star}^{\otimes \cdot} \rightarrow \Lambda \underline{\mathfrak{g}}_{2 \star}^{\otimes}$, where $d_{2 \star}=m_{2 \star}^{1}+m_{2}^{1,1}+\cdots$ whith $m_{2 \star}^{1}$ corresponding to the differential $b_{\star}=\left[m_{\star},-\right]_{G}$. We will also prove, following the same steps as in the $\pi=0$ case, that there exists a $G_{\infty}$-morphism between the $G_{\infty}$-algebras $\left(\mathfrak{g}_{1 \star}, d_{1 \star}\right)$ and $\left(\mathfrak{g}_{2 \star}, d_{2 \star}\right)$.

Theorem 7.1. - One can build a $G_{\infty}$-structure on $\mathfrak{g}_{2}[[\hbar]]$ determined by a differential $d_{2 \star}: \Lambda \underline{\mathfrak{g}_{2 \star}^{\otimes \cdot}} \rightarrow \Lambda \underline{\mathfrak{g}_{2 \star}^{\otimes}}$ with $d_{2 \star}=m_{2 \star}^{1}+m_{2 \star}^{1,1}+\cdots+m_{2 \star}^{p_{1}, \ldots, p_{n}}+\cdots$, where

$$
m_{2 \star}^{p_{1}, \ldots, p_{n}}: \underline{\mathfrak{g}_{2}[[\hbar]]^{\otimes p_{1}}} \wedge \cdots \wedge \underline{\mathfrak{g}_{2}[[\hbar]]^{\otimes p_{n}}} \rightarrow \underline{\mathfrak{g}_{2}[[\hbar]]^{\otimes p_{1}}} \wedge \cdots \wedge \underline{\mathfrak{g}_{2}[[\hbar]]^{\otimes p_{n}}},
$$

$m_{2 \star}^{1}=b_{\star}=\left[m_{\star},-\right]_{G}$ and $m_{2 \star}^{1,1}=m_{2}^{1,1}$ is the Gerstenhaber bracket.

Proof: We can use the same arguments as in Section 2. Thanks to Lemma 2.1, it is enough to define a differential Lie bialgebra structure on the cofree Lie coalgebra $L_{2 \star}=$ $\oplus \mathfrak{g}_{2}[[\hbar]]^{\otimes n}$. Etingof-Kazhdan's dequantization and quantization theorems can be used in the same way to prove it is enough to have a differential bialgebra structure on the cofree tensorial coalgebra $T_{2 \star}=\oplus \mathfrak{g}_{2}[[\hbar]]^{\otimes n}$ since the correspondance in Proposition 2.2 was given by universal formulas.

So we want now to define a bialgebra structure on $T_{2 \star}$ given by maps $a_{2 \star}^{n}$ and $a_{2 \star}^{p_{1}, p_{2}}$ such that $a_{2 \star}^{1}=b_{\star}$ and $a_{2 \star}^{1,1}$ is the product $\{-\mid-\}$ defined in Section 1. This can be done using braces as in the end of Section 2. This is because the braces inducing a bialgebra structure on $\mathfrak{g}_{2}$ are independent of the algebra structure on $\mathfrak{g}_{2}$. Thus a $G_{\infty}$-structure can be built on $\mathfrak{g}_{\star}$ with $m_{2 \star}^{p_{1}, \ldots, p_{n}}=0$ for $n>2$.

We can now state the analogue of Proposition 3.1.

Theorem 7.2. - There exist a $G_{\infty}$-structure on $\mathfrak{g}_{1}[[\hbar]]$ corresponding to a differential $d_{1 \star}^{\prime}: \Lambda \underline{\mathfrak{g}_{1 \star}^{\otimes}} \rightarrow \Lambda \underline{\mathfrak{g}_{1 \star}^{\otimes}}$ and a morphism of differential coalgebras

$$
\psi_{\star}:\left(\Lambda \underline{\mathfrak{g}_{1 \star}}, d_{1 \star}^{\prime}\right) \rightarrow\left(\Lambda \underline{\mathfrak{g}_{2 \star}}, d_{2 \star}\right)
$$

such that the induced map $\mathfrak{g}_{1} \rightarrow \mathfrak{g}_{2}$ is the Hochschild-Kostant-Rosenberg map.

Proof: We will follow the proof of Proposition 3.1 and use the same notations. Let us denote

$$
V_{i \star}^{(n)}=\sum_{k=0}^{n} \hbar^{k} V_{i}^{[n-k]} \quad \text { and } \quad V_{i \star}^{(\leq n)}=\sum_{k=0}^{n} V_{i \star}^{(k)} .
$$


There is a decomposition $d_{2 \star}=\sum_{k>0} \hbar^{k} d_{2 \star}^{\{k\}}$. We denote $d_{2 \star}^{\{k\} p_{1}, \ldots, p_{l}}: \mathfrak{g}_{2}^{p_{1}} \wedge \cdots \wedge$ $\mathfrak{g}_{2}^{p_{l}} \rightarrow \mathfrak{g}_{2}$ the components of $d_{2 \star}: \Lambda \underline{\mathfrak{g}}_{2}^{\otimes} \rightarrow \Lambda \underline{\mathfrak{g}}_{2}^{\otimes}$. Similarly, we denote $d_{2 \star}^{\{k\}[n]}$ the map from $\Lambda \underline{\mathfrak{g}_{2}^{\otimes \cdot}}$ to itself defined by

$$
d_{2 \star}^{\{k\}[n]}=\sum_{p_{1}+\cdots+p_{l}=n} d_{2 \star}^{\{k\} p_{1}, \ldots, p_{l}} .
$$

We have the obvious identity $d_{2 \star}^{\{k\}}=\sum_{n \geq 1} d_{2 \star}^{\{k\}[n]}$. We can now define

$$
d_{2 \star}^{(m)}=\sum_{k+n=m} d_{2 \star}^{\{k\}[n]}
$$

and set

$$
d_{2 \star}=\sum_{m \geq 1} d_{2 \star}^{(m)}, \quad d_{2 \star}^{(\leq m)}=\sum_{i=1}^{m} d_{2 \star}^{(i)} .
$$

In the same way we set

$$
\begin{aligned}
& d_{1 \star}^{\prime}=\sum_{m \geq 1} d_{1 \star}^{\prime(m)}, \quad d_{1 \star}^{\prime(m)}=\sum_{k+n=m} d_{1 \star}^{\prime}{ }^{\{k\}[n]}, \quad d_{1 \star}^{\prime}{ }^{(\leq m)}=\sum_{i=1}^{m} d_{1 \star}^{\prime(i)}, \\
& \psi_{\star}=\sum_{m \geq 1} \psi_{\star}{ }^{(m)}, \quad \psi_{\star}{ }^{(m)}=\sum_{k+n=m} \psi_{\star}{ }^{\{k\}[n]} \quad \text { and } \quad \psi_{\star}{ }^{(\leq m)}=\sum_{i=1}^{m} \psi_{\star}{ }^{(i)} .
\end{aligned}
$$

The proof of Proposition 3.1 can now be reproduced, formally replacing the superscripts [-] with (-). We can build maps $d_{1 \star}^{\prime}$ and $\psi_{\star}$ by induction setting $d_{1 \star}^{\prime(1)}=0$ and $\psi_{\star}^{(1)}=\phi^{1}$, the Hochschild-Kostant-Rosenberg map. The proof then relies again only on the fact that $\phi^{1}$ is a quasi-isomorphism of complexes from $\left(\mathfrak{g}_{1}, 0\right)$ to $\left(\mathfrak{g}_{2}, b=\right.$ $\left.m_{2}^{1}=m_{2 \star}^{(1)}\right)$ (for which we have homotopy formulas). Moreover, at order two we have again

$$
d_{1 \star}^{\prime(2)}=d_{1 \star}{ }^{(2)}=\hbar[\pi,-]_{S}+d_{1}^{1,1}+d_{1}^{2} .
$$

Using the grading (-) along the lines of the proof of Theorem 4.1, we can prove in the same way the following.

Theorem 7.3. - If the complex $\left(\operatorname{End}\left(\Lambda \underline{\mathfrak{g}}_{1}^{\otimes \cdot}\right),\left[m_{1}^{1,1}+m_{1}^{2}+\hbar[\pi,-]_{S},-\right]\right)$ is acyclic, then there exists a $G_{\infty}$-morphism $\psi^{\prime}:\left(\Lambda \underline{\mathfrak{g}_{1 \star}}, d_{1 \star}\right) \rightarrow\left(\Lambda \underline{\mathfrak{g}_{1 \star}^{\otimes}}, d_{1 \star}^{\prime}\right)$ such that the induced map $\mathfrak{g}_{1} \rightarrow \mathfrak{g}_{2}$ is the identity.

Theorems 7.2 and 7.3 hold for arbitrary Poisson manifolds.

Corollary 7.4. - If $\mathfrak{g}_{1}=\Gamma\left(\mathbb{R}^{m}, \Lambda T \mathbb{R}^{m}\right)$, there exists a $G_{\infty}$-morphism

$$
\psi^{\prime}:\left(\Lambda \underline{\mathfrak{g}_{1 \star}^{\otimes}}, d_{1 \star}\right) \rightarrow\left(\Lambda \underline{\mathfrak{g}_{1 \star}^{\otimes}}, d_{1 \star}^{\prime}\right) .
$$


Proof: Using Theorem 7.3 it is enough to check that the cochain complex

$$
\left(\operatorname{Hom}\left(\Lambda \underline{\mathfrak{g}_{1}^{\otimes}}, \Lambda \underline{\mathfrak{g}_{1}^{\otimes \cdot}}\right),\left[m_{1}^{1,1}+m_{1}^{2}+\hbar[\pi,-]_{S},-\right]\right)
$$

is for $M=\mathbb{R}^{m}$. This follows from Proposition 5.1 and the following Lemma 7.5.

Lemma 7.5. - If a complex $\left(C, d_{0}\right)$ is acyclic, then, for any differential $d_{\star}=d_{0}+$ $\sum_{i \geq 1} \hbar^{i} d_{i}$, the $\mathbb{R}[[\hbar]]$-linear complex $\left(C[[\hbar]], d_{0}+\sum_{i \geq 1} \hbar^{i} d_{i}\right)$ is acyclic.

This follows from Remark 6.3. Howewer, as we wish to be able to construct explicit homotopies we give another proof.

Proof: Suppose we have $x=\sum_{i \geq 0} \hbar^{i} x_{i} \in C[[\hbar]]$ satisfying

$$
d_{\star} x=0 .
$$

We will construct by induction $y=\sum_{i \geq 0} \hbar^{i} y_{i}$ satisfying $x=d_{\star} y$.

Relation (7.18) at order 0 gives $d_{0} x_{0}=0$; so by hypothesis there exists $y_{0} \in C$ such that $x_{0}=d_{0} y_{0}$. Suppose we have built $y_{i}$ for $i \leq n-1$ such that $x_{k}=\sum_{i=0}^{k} d_{i} y_{k-i}$ for all $k \leq n-1$. We want to build $y_{n}$ such that $x_{n}=\sum_{i=0}^{n} d_{i} y_{n-i}$. From the acyclicity of the complex $\left(C, d_{0}\right)$ this is equivalent to

$$
d_{0}\left(x_{n}-\sum_{i=1}^{n} d_{i} y_{n-i}\right)=0 .
$$

We have

$$
\Longleftrightarrow \sum_{i=1}^{n} d_{i} x_{n-i}+\sum_{i=1}^{n} d_{0} d_{i} y_{n-i}=0
$$

By the induction hypothesis, we obtain

$$
\begin{aligned}
& \Longleftrightarrow \sum_{i=1}^{n} d_{i} x_{n-i}-\sum_{i=1}^{n} \sum_{j=1}^{i} d_{j} d_{i-j} y_{n-i}=0 \\
& \Longleftrightarrow \sum_{i=1}^{n} d_{i} x_{n-i}-\sum_{j=1}^{n} d_{j} \sum_{i=j}^{n} d_{i-j} y_{n-i}=0 \\
& \Longleftrightarrow \sum_{i=1}^{n} d_{i} x_{n-i}-\sum_{j=1}^{n} d_{j} \sum_{i=0}^{n-j} d_{i} y_{n-i-j}=0 \\
& \Longleftrightarrow \sum_{i=1}^{n} d_{i} x_{n-i}-\sum_{j=1}^{n} d_{j} x_{n-j}=0 .
\end{aligned}
$$

This proves the result.

As in Section 6, it is easy to see that $\phi_{\star}=\psi_{\star}^{\prime} \circ \psi_{\star}$ is a $G_{\infty}$-morphism between $\mathfrak{g}_{1 \star}$ and $\mathfrak{g}_{2 \star}$. Moreover $\phi_{\star}$ restricts to a $L_{\infty}$-morphism

$$
\widetilde{\phi_{\star}}:\left(\mathfrak{g}_{1}[[\hbar]], \hbar[\pi,-]_{S},[-,-]_{S}\right) \rightarrow\left(\mathfrak{g}_{2}[[\hbar]], b_{\star},[-,-]_{G}\right)
$$


and also to a $A_{\infty}$-morphism

$$
\check{\phi}_{\star}:\left(\mathfrak{g}_{1}[[\hbar]], \hbar[\pi,-]_{S}, \wedge\right) \rightarrow\left(\mathfrak{g}_{2}[[\hbar]], b_{\star}, \sum_{i \geq 2} m_{2 \star}^{i}\right) .
$$

If we now restrict the map $\phi_{\star}$ to $\phi_{\star}^{[1]}: \mathfrak{g}_{1}[[\hbar]] \rightarrow \mathfrak{g}_{1}[[\hbar]]$, we have $\phi_{\star}^{[1]}\left([\hbar \pi, \alpha]_{S}\right)=$ $b_{\star} \phi_{\star}^{[1]}(\alpha)$ for any $\alpha \in \mathfrak{g}_{1}$. So we have constructed another morphism of complexes $\left(\mathfrak{g}_{1}[[\hbar]], \hbar[\pi,-]_{S}\right) \rightarrow\left(\mathfrak{g}_{2}[[\hbar]], b_{\star}\right)$. According to Lemma 6.2 , the map $\phi_{\star}^{[1]}$ is a quasiisomorphism which is a priori different from the map $\phi_{\hbar}^{1}$. We leave the two following questions unanswered:

Question 1: Are the two maps $\phi_{\hbar}^{1}$ and $\phi_{\star}^{[1]}$ the same?

Question 2: To prove the existence of the $G_{\infty}$-morphism $\phi_{\star}$, we have used the grading (-) which imposes the initial condition $\psi_{\star}^{(1)}=\phi^{1}$, the Hochschild-KostantRosenberg morphism. Is it possible to build a map $\phi_{\star}$ such that $\phi_{\star}^{[1]}=\phi_{\hbar}^{1}$ ?

Remark: B. Keller helped us to give a partial answer to this second question, using the following proposition (see K. Lefvre [Le] for a proof in the $A_{\infty}$ case).

Proposition 7.6. - Let $A$ and $B$ be two $L_{\infty}$ (respectively $A_{\infty}$, or $G_{\infty}$ )-algebras, with structures determined by differentials

$$
d_{A}: \Lambda^{\prime} A \rightarrow \Lambda^{\prime} A \text { (respectively } \underline{A^{\otimes \cdot}} \rightarrow \underline{A^{\otimes \cdot}} \text { or } \Lambda^{\cdot} \underline{A^{\otimes \cdot}} \rightarrow \Lambda^{\cdot} \underline{A^{\otimes \cdot}} \text { ), }
$$

and $d_{B}$ defined in the same way. Denote $d_{A}=\sum_{n \geq 0} d_{A}^{n}(; \ldots$,$) , where d_{A}^{n}$ is a homogeneous component of $d_{A}$ (in the $G_{\infty}$ case, we write $d_{A}=\sum_{l \geq 0, n_{1}+\cdots+n_{p}=l} d_{A}^{n_{1}, \ldots, n_{p}}$ whith $d_{A}^{n_{1}, \ldots, n_{p}}: \underline{A^{\otimes n_{1}}} \Lambda \ldots \Lambda \underline{A^{\otimes n_{p}}} \rightarrow B$ and we order the maps $d_{i}^{n_{1}, \ldots, n_{p}}$ such that $\left(n_{1}, \ldots, n_{p}\right) \geq\left(m_{1}, \ldots, m_{q}\right) \Leftrightarrow\left(n_{1}+\cdots+n_{p}>m_{1}+\cdots+m_{q}\right)$ or $\left(n_{1}+\cdots+n_{p}=\right.$ $m_{1}+\cdots+m_{q}$ and $\left(n_{1}, \ldots, n_{p}\right) \geq\left(m_{1}, \ldots, m_{q}\right)$ for the lexicographic order). Suppose there exists a "twisting" element $a \in A$ such that

$$
\sum_{n \geq 0} d_{A}^{n}(a, \ldots, a)=0
$$

and a $L_{\infty}$ (respectively $A_{\infty}$, or $G_{\infty}$ )-morphism $\varphi=\sum_{n \geq 0} \varphi^{n}$ (using the same convention as above) between $A$ and $B$. Then

: $(a)=$ there exists a "twisted" $L_{\infty}$ (respectively $A_{\infty}$, or $\left.G_{\infty}\right)$-algebra structure on $A$ with differential $d_{A_{a}}=\sum_{n \geq 0} d_{A_{a}}^{n}$ given by

$$
d_{A_{a}}^{n}(\cdot, \ldots, \cdot)=\sum_{i \geq 0} d_{A}^{n+i}(\ldots, a, \ldots, a, \ldots),
$$

where the element $a$ is inserted $i$ times;

: (b) the element $b \in B$ defined by

$$
b=\sum_{n \geq 0} \varphi^{n}(a, \ldots, a)
$$

satisfies

$$
\sum_{n \geq 0} d_{B}^{n}(b, \ldots, b)=0 .
$$


: (c) there exists a "twisted" $L_{\infty}$ (respectively $A_{\infty}$, or $G_{\infty}$ )-algebra structure on $B$ with differential $d_{B_{b}}=\sum_{n \geq 0} d_{B_{b}}^{n}$ given by

$$
d_{B_{b}}^{n}(\cdot, \ldots, \cdot)=\sum_{i \geq 0} d_{B}^{n+i}(\ldots, b, \ldots, b, \ldots)
$$

where the element $b$ is inserted $i$ times;

: (d) there exists a $L_{\infty}$ (respectively $A_{\infty}$, or $G_{\infty}$ )-morphism between the two "twisted" $L_{\infty}$ (respectively $A_{\infty}$, or $G_{\infty}$ )-algebra structures on $A$ and $B$ given by $\varphi_{a b}=\sum_{n \geq 0} \varphi_{a b}^{n}$, where

$$
\varphi_{a b}^{n}(\cdot, \ldots, \cdot)=\sum_{i \geq 0} \varphi^{n+i}(\ldots, a, \ldots, a, \ldots)
$$

where the element $a$ is inserted $i$ times.

In our case, where $A=\mathfrak{g}_{1}$ and $B=\mathfrak{g}_{2}$ and $\varphi$ is Tamarkin's $L_{\infty}$ (respectively $A_{\infty}$, or $G_{\infty}$ )-morphism, and $a=\pi$ (the Poisson tensor field), we can apply the previous proposition, but only in the $L_{\infty}$ case (because otherwise $\sum d_{1}^{k}(\pi, \ldots, \pi) \neq 0$ ), and get a deformed $L_{\infty}$-morphism between the graded Lie algebras $\left(\mathfrak{g}_{1}[[\hbar]], \hbar[\pi,-]_{S},[-,-]_{S}\right)$ and $\left(\mathfrak{g}_{2}[[\hbar]], b_{\star},[-,-]_{G}\right)$.

\section{ACKNOWLEDGEMENTS}

We would like to thank D. Manchon and B. Keller for many useful suggestions. We also would like to thank C. Kassel for a carefuly reading of the paper.

\section{References}

[Ba] J. H. Baues, The double bar and cobar constructions, Compos. Math. 43 (1981), 331-341 [BFFLS1] F. Bayen, M. Flato, C. Fronsdal, A. Lichnerowicz, D. Sternheimer, Quantum mechanics as a deformation of classical mechanics, Lett. Math. Phys. 1 (1975/77), no. $6,521-530$

[BFFLS2] F. Bayen, M. Flato, C. Fronsdal, A. Lichnerowicz, D. Sternheimer, Deformation theory and quantization, I and II, Ann. Phys. 111 (1977), 61-151

[CE] H. Cartan, S. Eilenberg, Homological algebra, Princeton University Press, Princeton, N. J. (1956)

[CFT] A. S. Cattaneo, G. Felder, L. Tomassini, Fedosov connections on jet bundles and deformation quantization, math.QA/0111290 (to appear in IRMA Lecture Notes in Mathematics and Theoretical Physics : Deformation Quantization - Proceedings of the Meeting between Mathematicians and Theoretical Physicists, Institut de Recherche Mathématique Avancée de Strasbourg (May 31 - June 2, 2001)

[DL] M. De Wilde, P. B. A. Lecomte, A homotopy formula for the Hochschild cohomology, Compositio Math. 96 (1995), no. 1, 99-109

[EK1] P. Etingof, D. Kazhdan, Quantization of Lie bialgebras. I, Selecta Math. (N.S.) 2 (1996), no. 1, 1-41 
[EK2] P. Etingof, D. Kazhdan, Quantization of Lie bialgebras. II, III, Selecta Math. (N.S.) 4 (1998), no. 2, 213-231, 233-269

[GV] M. Gerstenhaber, A. Voronov, Homotopy G-algebras and moduli space operad, Internat. Math. Res. Notices (1995), no. 3, 141-153

[Gi] G. Ginot, Homologie et modèle minimal des algèbres de Gerstenhaber, Preprint Institut de Recherche Mathématique Avancée, Strasbourg (2001), no. 42

[GK] V. Ginzburg, M. Kapranov, Koszul duality for operads, Duke Math. J. 76 (1994), No $1,203-272$

[Ha] G. Halbout, Formule d'homotopie entre les complexes de Hochschild et de de Rham, Compositio Math. 126 (2001), No 2, 123-145

[Hi] V. Hinich, Tamarkin's proof of Kontsevich's formality theorem, math.QA/9803025

[HKR] G. Hochschild, B. Kostant and A. Rosenberg, Differential forms on regular affine algebras, Transactions AMS 102 (1962), 383-408

[Ka] C. Kassel, Homologie cyclique, caractère de Chern et lemme de perturbation, J. Reine Angew. Math. 408 (1990), 159-180

[Kh] M. Khalkhali, Operations on cyclic homology, the $X$ complex, and a conjecture of Deligne, Comm. Math. Phys. 202 (1999), no.2, 309-323

[Ko1] M. Kontsevich, Formality conjecture. Deformation theory and symplectic geometry, Math. Phys. Stud. 20, Ascona (1996), 139-156

[Ko2] M. Kontsevich, Deformation quantization of Poisson manifolds, I, q-alg/9709040

[KS] M. Kontsevich, Y. Soibelman, Deformations of algebras over operads and the Deligne conjecture, Confrence Mosh Flato 1999, Vol. I (Dijon) Math. Phys. Stud. 21 (2000), $255-307$

[Kos] J. L. Koszul, Crochet de Schouten-Nijenjuis et cohomologie in "Elie Cartan et les mathémati-ques d'aujourd'hui", Astérisque (1985), 257-271

[LS] T. Lada, J. D. Stasheff, Introduction to SH Lie algebras for physicists, Internat. J. Theoret. Phys. 32 (1993), No 7, 1087-1103

[Le] K. Lefèvre, Sur les $A_{\infty}$-catégories, Thesis, available at http://www.institut.math.jussieu.fr/ lefevre/

[Lo1] J.-L. Loday, Cyclic homology, Springer Verlag (1993)

[Lo2] J.-L. Loday, Opérations sur l'homologie cyclique des algèbres commutatives, Invent. Math. 96 (1989), No. 1, 205-230

[St] J.D. Stasheff, Homotopy associativity of $H$-spaces I, II, Trans. Amer. Math. Soc. 108 (1963), 275-292

[Ta] D. Tamarkin, Another proof of M. Kontsevich's formality theorem, math.QA/9803025

[TS] D. Tamarkin, B Tsygan, Noncommutative differential calculus, homotopy BV algebras and formality conjectures, Methods Funct. Anal. Topology, 6 (2000), no. 2, 85-97

[Vo] A. Voronov, Homotopy Gerstenhaber algebras, Conférence Mosh Flato 1999, Vol. II (Dijon), Math. Phys. Stud., 22, Kluwer Acad. Publ. (2000), 307-331

GrEgory Ginot, Institut de Recherche Mathématique Avancée, Université Louis Pasteur - C.N.R.S., 7, rue René Descartes, 67084 Strasbourg Cedex, France $\bullet$ E-mail : ginot@math.u-strasbg.fr

Gilles Halbout, Institut de Recherche Mathématique Avancée, Université Louis Pasteur C.N.R.S., 7, rue René Descartes, 67084 Strasbourg Cedex, France $\bullet E$-mail : halbout@math.u-strasbg.fr 